\title{
O Efeito da Carga Hidrológica e sua Influência no Deslocamento e nas Velocidades de Estações GNSS na Bacia Amazônica Brasileira
}

\author{
The Effect of Hydrological Load and its Influence on the Displacement and Velocities of \\ GNSS Stations in the Brazilian Amazon Basin
}

Lécio Alves Nascimento ${ }^{1}$, William Rodrigo Dal Poz ${ }^{2}$ e Krisley Xavier Soares de Freitas ${ }^{3}$

1 Instituto Federal de Educação, Ciência e Tecnologia do Norte de Minas Gerais (IFNMG) -Campus Araçuaí -MG, Brasil, lecio.nascimento@ifnmg.edu.br

ORCID: https://orcid.org/0000-0003-2499-4372

2 Universidade Federal de Viçosa (UFV), Departamento de Engenharia Civil, Viçosa -MG, Brasil, william.dalpoz@ufv.br ORCID: https://orcid.org/0000-0001-9532-3643

3 Universidade Federal de Viçosa (UFV), Departamento de Engenharia Civil, Viçosa -MG, Brasil, krisley.freitas@ufv.br ORCID: https://orcid.org/0000-0001-8453-324X

Resumo: Fenômenos geodinâmicos e de deformação crustal alteram as posições de pontos na superfície terrestre. Alguns destes, como a carga hidrológica (water storage e hidrological load), tem expressividade em regiões específicas, como a Amazônica, consistindo-se como uma característica local. Seu efeito se manifesta como deformações viscoelásticas temporais na superfície terrestre e não são considerados na modelagem do PPP (Posicionamento por Ponto Preciso). Nesse sentido, o presente trabalho objetiva investigar a influência da carga hidrológica no deslocamento de nove estações da RBMC (AMBC, AMCO, AMHA, AMMU, AMTE, AMUA, ITAM, NAUS e PAIT) situadas na Bacia Amazônica, utilizando séries temporais posicionais geradas no IBGE-PPP. As séries de componentes verticais $(\Delta u)$ apresentaram maiores amplitudes, quando comparadas às séries de componentes norte $(\Delta n)$ e este $(\Delta e)$. A estação NAUS apresentou maior amplitude média para série $\Delta u(0,073 \mathrm{~m})$ e amplitude máxima em $2012(0,084 \mathrm{~m})$, justificável pela maior cheia histórica. As séries $\Delta n$ apresentaram resultados similares à série $\Delta u$, porém com amplitudes menores. A estação NAUS obteve amplitude máxima $(0,023 \mathrm{~m})$ em 2012 e 2015 para série $\Delta n$, justificável pelas cheias, e amplitude média de $0,020 \mathrm{~m}$. Em relação às séries $\Delta e$, maior amplitude média $(0,016$ $\mathrm{m})$ foi obtida por AMBC. Comprovado o efeito da carga hidrológica nas componentes posicionais, comparou-se as velocidades derivadas das séries e do VEMOS2009, em que Vn, Ve e Vu apresentaram discrepâncias variando entre $-0,0001$ e $0,0014 \mathrm{~m}$; -0,0021 e 0,0017; e -0,0027 e 0,0084 respectivamente.

Palavras-chave: Geodésia. Carga hidrológica. Velocidade. GNSS. PPP.

Abstract: Crustal deformation and Geodynamic phenomena alter the positions of points on the Earth's surface. Some of these, such as the hydrological load (water storage and hydrological load), are expressive in specific regions, such as the Amazon basin, becoming to consist of yourself of a local characteristic. Its effect is manifested as temporal viscoelastic deformations on the Earth's surface and are not considered in the PPP (Precise Point Positioning) modeling. In this sense, the present work aims to investigate the influence of the hydrological load in the displacement of nine stations of the RBMC (AMBC, AMCO, AMHA, AMMU, AMTE, AMUA, ITAM, NAUS and PAIT) located in the Amazon Basin, using positional time series generated in the IBGE-PPP. The series of vertical components $(\Delta u)$ presented greater amplitudes when compared to the series of the north $(\Delta n)$ and east $(\Delta e)$ components. The NAUS station presented the highest average amplitude for the $\Delta u$ series $(0.073 \mathrm{~m})$ and the maximum amplitude in 2012 $(0.084 \mathrm{~m})$, justified by the largest historical flood. The $\Delta n$ series presented results similar to the $\Delta u$ series, but with smaller amplitudes. The NAUS station achieved maximum amplitude $(0.023 \mathrm{~m})$ in 2012 and 2015 for the $\Delta \mathrm{n}$ series, justified by the floods, and an average amplitude of $0.020 \mathrm{~m}$. In relation to the $\Delta e$ series, the largest mean amplitude $(0.016 \mathrm{~m})$ was obtained by AMBC. After proving the effects of the hydrological load on the positional components, the velocities derived from the time series and VEMOS2009 were compared, in which Vn, Ve, and $\mathrm{V} u$ presented discrepancies between $-0,0001$ and $0,0014 \mathrm{~m},-0,0021$ and 0.0017 , and -0.0027 and 0.0084 respectively.

Keywords: Geodesy. Hydrological load. Velocity. GNSS. PPP. 


\section{INTRODUÇÃO}

Fenômenos geodinâmicos e de deformação crustal provocam deslocamentos ao longo do tempo, causando alterações nas posições espaciais (coordenadas) de pontos da superfície terrestre (SAPUCCI; MONICO, 2001; SANCHEZ; DREWES, 2016).

Diversos destes fenômenos são investigados fundamentando-se nas alterações posicionais ao longo do tempo, tais como: o movimento de placas tectônicas (ALINIA; TIAMPO; JAMES, 2017; KOWALCZYK; RAPINSKI, 2018), a atividade vulcânica (GEIRSSON et al. 2017), o derretimento de geleiras (RAJNER, 2018) e a carga hidrológica (BEVIS et al., 2005; FRITSCHE; DOLL; DIETRICH, 2012; NASCIMENTO, 2016).

Comumente, emprega-se em tais investigações séries temporais posicionais geradas a partir de dados GNSS (Global Navigation Satellite System) advindos das redes ativas de monitoramento contínuo, pósprocessados em serviços de PPP (Posicionamento por Ponto Preciso) online, o que se justifica em virtude: da gratuidade, da quantidade e qualidade dos dados, da acurácia alcançada nos serviços (centimétrica) etc. (GRINTER; ROBERTS, 2011; CHOY; BISNATH; RIZOS, 2016). Adicionalmente, evidencia-se que as posições estimadas no PPP independem de estações de referência, condição essencial aos estudos que demandem variações locais das posições (MOREIRA, 2010).

Nesse sentido, salienta-se que efeitos de diversos fenômenos são modelados no PPP, conforme recomendações de Petit e Luzum (2010). No entanto, o efeito da carga hidrológica (water storage e hidrological load) é desconsiderado no PPP por não apresentar modelagem consistente em escala global (PETIT; LUZUM, 2010; RAJNER; LIWOSZ, 2012). Tal efeito é representativo em regiões específicas que apresentam transferência significativa de massa d'água, como a Amazônica, derivando de um processo hidrogeodinâmico associado ao ciclo hidrológico, que ocasiona deformações viscoelásticas temporais (transitórias) na superfície da Terra (TREGONING et al., 2009; FRITSCHE; DOLL; DIETRICH, 2012).

Estudos afins ou correlatos, associados à influência do efeito da carga hidrológica na componente altimétrica são comumente encontrados, como em: Van Dam et al. (2001), Romagnoli et al. (2003), Davis et al. (2004), Bevis et al. (2005), Rosa (2008), Costa, Matos e Blitzkow (2012), Moreira et al. (2016), Nascimento (2016), Giacometti et al. (2017), Oliveira (2018) e Knowles, Bennett e Harig (2020). No entanto, há necessidade de mais estudos que investiguem a influência deste efeito nas componentes planimétricas, como os apresentados por Fu et al. (2013) e Azambuja e Matsuoka (2016), uma vez que podem degradar produtos derivados, como as velocidades (deformação contínua da superfície) das estações GNSS.

SIRGAS (2020) recomenda que as velocidades devem advir de levantamentos GNSS contínuos ou repetitivos (séries temporais) que cubram um período mínimo de dois anos e, caso isso não seja possível, sejam extraídas de um modelo de velocidades ou deformações. No entanto, não apresenta diretrizes ou recomendações quanto aos critérios e/ou metodologias voltadas à utilização de séries temporais. Nesse sentido, Bogusz e Klos (2016) e Olds et al. (2019) destacam que a determinação das velocidades a partir das séries temporais de posição é comumente efetuada com o ajuste do modelo de regressão linear simples.

Salienta-se que no Brasil utiliza-se frequentemente o VEMOS2009 (modelo de velocidade para o SIRGAS 2009) (DREWES; HEIDBACH, 2012) para a determinação das velocidades. As velocidades são amplamente empregadas nos procedimentos de atualização/redução de coordenadas, como o utilizado pelo serviço online IBGE-PPP (Instituto Brasileiro de Geografia e Estatística-PPP) (IBGE, 2017).

Em vista do exposto, propõe-se investigar as consequências do efeito da carga hidrológica nas séries temporais de componentes posicionais e sua influência na determinação das velocidades de estações da RBMC situadas na Bacia Amazônica, fundamentando-se no ajuste do modelo de regressão linear. Adicionalmente, o efeito da compatibilização entre as materializações do ITRF (International Terrestrial Reference Frame) (IGS - International GNSS Service) e suas conversões para o SIRGAS2000 (Sistema de Referência Geocêntrico para as Américas 2000) nas séries temporais posicionais serão analisados.

\section{GERAÇÃO DAS SÉRIES TEMPORAIS POSICIONAIS}

Define-se uma série temporal posicional como um conjunto de valores de posições (coordenadas) 
associadas a um sistema de referência e ordenadas no tempo. Dentre os mecanismos utilizados para se obter/construir uma série temporal posicional tem-se o RINEX EDITION (NASCIMENTO et al., 2017), que consiste em um programa computacional que viabiliza informações posicionais (coordenadas cartesianas geocêntricas $X, Y, Z$; elipsoidais $\varphi, \lambda, h$ e as precisões de ambas) a partir de dados GNSS das estações da RBMC (COSTA et al., 2018; RBMC, 2020) em conjunto com o serviço online IBGE-PPP (IBGE-PPP, 2020). A edição dos arquivos RINEX utiliza o TEQC (Translation, Editing and Quality Check) (ESTEY; MEERTENS, 1999; UNAVCO, 2020). Destaque será dado a este por ser utilizado neste trabalho.

O IBGE-PPP consiste em uma adaptação do CSRS-PPP (NRCAN, 2020) (versão 1.05/11216 atualizada) para uso no Brasil e fornece resultados acurados tanto na materialização do IGS referente à época de coleta dos dados (atualmente IGb14, compatível e alinhado com o ITRF2014) quanto no SIRGAS2000. Neste último disponibiliza-se as coordenadas também na data de realização do sistema, época 2000,4 (IBGE, 2017; IBGE-PPP, 2020). Salienta-se que o SIRGAS2000 é o sistema de referência oficial brasileiro e deve ser adotado em todas as atividades de informações geoespaciais (IBGE, 2015; INDE, 2020). Salienta-se que os parâmetros de processamento utilizados no IBGE-PPP são padronizados e podem ser consultados em IBGE (2017).

Originalmente, as coordenadas estimadas no IBGE-PPP estão referenciadas ao ITRF (IGS) (sistema utilizado nas órbitas IGS), época de coleta dos dados, cujas realizações são apresentadas na Tabela 1.

Tabela 1 - Soluções IGS adotadas pelo IBGE-PPP.

\begin{tabular}{c|c}
\hline Data de rastreio GNSS & Realização IGS \\
\hline 05/11/2006 a 16/04/2011 & IGS05 \\
$17 / 04 / 2011$ a 06/10/2012 & IGS08 \\
$07 / 10 / 2012$ a 28/01/2017 & IGb08 \\
$29 / 01 / 2017$ a 16/05/2020 & IGS14 \\
$17 / 05 / 2020$ a atualmente & IGb14 \\
\hline
\end{tabular}

Fonte: IBGE (2017) e IBGE-PPP (2020).

As realizações utilizadas pelo IGS em seus produtos são alinhadas e compatíveis com as materializações dos ITRFs, compartilhando a mesma origem, escala e orientação (IGSACC, 2020). Destacase que o IGb08 é uma atualização do IGS08, assim como o IGb14 é uma atualização do IGS14 e visam estabilizar o alinhamento dos referenciais dos produtos IGS, sem alterações aos usuários (REBISCHUNG, 2020a; REBISCHUNG, 2020b). Maiores informações sobre as materializações ITRF (IGS) em IGSACC (2020).

Altamimi et al. (2016) destacam que a materialização do ITRF2014 (IGS14) apresenta maior consistência em relação as anteriores devido à consideração dos movimentos não lineares das estações, incluindo sinais sazonais (anuais e semestrais) de posições de estações e deformação ocorridas após eventos sísmicos causados por grandes terremotos. De forma análoga, encontra-se em desenvolvimento o ITRF2020, cujas soluções finais tem previsão de divulgação entre os meses de setembro e outubro de 2021 (ITRF, 2020).

Nesse sentido, as séries temporais geradas no IBGE-PPP podem conter mais de uma materialização ITRF (IGS), a depender do período de abrangência. Em alguns casos, pode ser necessário efetuar a compatibilização entre as materializações de origem e destino, em suas respectivas épocas. O procedimento para a execução da compatibilização consiste, inicialmente, na atualização dos parâmetros (três translações, um fator de escala e três rotações) de sua época $\left(t_{k}\right)$ para a época de coleta dos dados $\left(t_{o}\right)$, caso sejam disponibilizadas as variações temporais destes parâmetros e, na sequência, aplicá-los na transformação de Helmert. Salienta-se que tanto os parâmetros quanto as coordenadas consideradas nas materializações devem estar na mesma época $t_{o}$. Destaca-se que tal procedimento é efetuado sob as componentes cartesianas geocêntricas $X, Y, Z$. Detalhes dos procedimentos para a compatibilização entre as materializações ITRF (IGS) aplicados neste trabalho podem ser consultados em Petit e Luzum (2010), Carvalho, Dal Poz e Larocca (2015), Ramos, Dal Poz e Carvalho (2016), Braga e Dal Poz (2019) dentre outros.

Considerando a complexidade associada à interpretação de resultados atrelados às componentes cartesianas geocêntricas $X, Y$ e $Z$, Leick (2004) destaca que tais resultados são mais facilmente interpretados 
quando estas são transformadas para o SGL (Sistema Geodésico Local). Ademais, o SGL possibilita avaliar as variações locais específicas, ocorridas em $X, Y$ e $Z$, em termos das componentes horizontais e vertical (MONICO, 2008).

Nesse sentido, o relacionamento entre as componentes cartesianas geocêntricas $X, Y$ e $Z$ e as componentes norte $(\Delta n)$, leste $(\Delta e)$ e vertical $(\Delta u)$ do SGL se dá a partir de uma transformação específica, em que a origem e a orientação espacial do SGL são dependentes das componentes elipsoidais $\varphi_{o}$ (latitude) e $\lambda_{o}$ (longitude) do ponto de origem (LEICK, 2004; HOFMANN-WELLENHOF; LICHTENEGGER; WASLE, 2008). No caso das séries temporais utiliza-se as componentes elipsoidais médias como origem, ou seja, $\left(\varphi_{o}, \lambda_{o}\right)=(\bar{\varphi}, \bar{\lambda})$ (SILVA; MONICO, 2012; MACIUK; SZOMBARA, 2018). Nesse contexto, as séries temporais de componentes geodésicas locais $(\Delta n, \Delta e, \Delta u)$ são determinadas com a aplicação da Eq. (1):

$$
\left[\begin{array}{l}
\Delta n_{j} \\
\Delta e_{j} \\
\Delta u_{j}
\end{array}\right]=\left[\begin{array}{ccc}
-\operatorname{sen} \bar{\varphi} \cos \bar{\lambda} & -\operatorname{sen} \bar{\varphi} \operatorname{sen} \bar{\lambda} & \cos \bar{\varphi} \\
-\operatorname{sen} \bar{\lambda} & \cos \bar{\varphi} & 0 \\
\cos \bar{\varphi} \cos \bar{\lambda} & \cos \bar{\varphi} \operatorname{sen} \bar{\lambda} & \operatorname{sen} \bar{\varphi}
\end{array}\right] *\left[\begin{array}{c}
X_{j}-\bar{X} \\
Y_{j}-\bar{Y} \\
Z_{j}-\bar{Z}
\end{array}\right]
$$

em que: $\left(\Delta n_{j}, \Delta e_{j}, \Delta u_{j}\right)$ são as componentes geodésicas locais da $j$-ésima época de interesse; $\left(X_{j}, Y_{j}, Z_{j}\right)$ são as componentes cartesianas da $j$-ésima época de interesse; $(\bar{X}, \bar{Y}, \bar{Z})$ são as componentes cartesianas médias das séries temporais; e, $(\bar{\varphi}, \bar{\lambda})$ são as componentes elipsoidais médias referentes às componentes cartesianas médias das séries temporais.

Salienta-se que as séries temporais de componentes geodésicas locais são essenciais ao desenvolvimento deste trabalho.

\section{REGRESSÃO LINEAR SIMPLES E DETERMINAÇÃO DE VELOCIDADES}

Kazmier (2007) e Bussab e Morettin (2012) evidenciam que os valores dos coeficientes de regressão linear $b_{0}$ e $b_{1}$ são estimados baseando-se nos dados amostrais de $N$ observações, cuja equação de regressão linear amostral é definida conforme Eq. (2):

$$
\widehat{Y}_{i}=Y_{i}-\varepsilon_{i}=b_{0}+b_{1} X_{i}
$$

em que: $\widehat{Y}_{i}$ indica o valor estimado (ou ajustado) para a $i$-ésima observação da variável dependente; $Y_{i}$ é a variável dependente (ou de resposta) na $i$-ésima observação; $\varepsilon_{i}$ é o erro aleatório (resíduo) em $Y_{i}$ para a $i$-ésima observação; $b_{0}$ é o intercepto de $\widehat{Y}_{i}$ para a população; $b_{1}$ é a inclinação da população, chamado de coeficiente de regressão; $X_{i}$ é a variável independente (ou explanatória) para a $i$-ésima observação; e $i=1,2,3, \ldots, N$.

Um dos métodos utilizados para determinar os valores de $b_{0}$ e $b_{1}$ é o MMQ (Método dos Mínimos Quadrados). Pelo critério do MMQ o melhor ajuste da linha de regressão (e consequentemente, a melhor equação) é aquele para o qual a soma dos desvios quadráticos entre os valores "real" e estimado $\left(\sum_{i=1}^{n}\left(\varepsilon_{i}\right)^{2}=\right.$ $\left.\sum_{i=1}^{n}\left(Y_{i}-\hat{Y}_{i}\right)^{2}\right)$ da variável dependente é minimizado para a amostra de dados considerada (KAZMIER, 2007; LEVINE et al., 2012).

Bogusz e Klos (2016), Fazilova, Ehgamberdiev e Kuzin (2018) e Olds et al. (2019) ressaltam que as componentes de velocidade $\vec{V}=\left(V_{n}, V_{e}, V_{u}\right)$ correspondem ao coeficiente $b_{1}$ da equação de regressão linear amostral, ajustada para cada série temporal posicional do SGL. O relacionamento destas componentes com as componentes do vetor velocidade nas coordenadas geodésicas elipsoidais $\vec{V}=\left(V_{\varphi}, V_{\lambda}, V_{h}\right)$ e cartesianas geocêntricas $\vec{V}=\left(V_{X}, V_{Y}, V_{Z}\right)$ pode ser verificado em Carvalho (2015).

\section{MATERIAIS E MÉTODOS}

Para a realização deste experimento foram selecionadas nove estações da RBMC situadas na Bacia Amazônica, cuja identificação e distribuição geoespacial é apresentada na Figura 1. Os períodos considerados 
bem como o quantitativo de dados correspondentes às séries temporais posicionais geradas, cuja data final é 31/12/2019 (dia do ano $n^{\circ} 365$ ), são apresentados na Tabela 2 para as respectivas estações.

Figura 1 - Identificação e geoespacialização das estações da RBMC utilizadas.

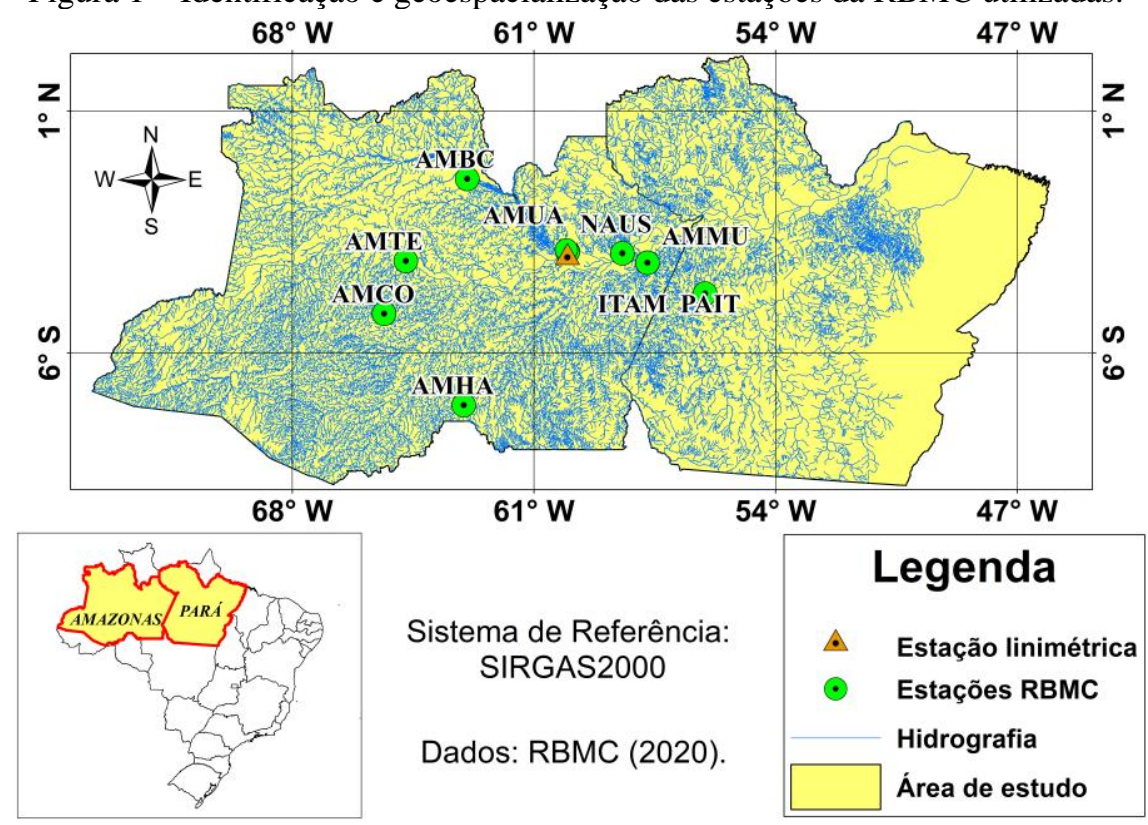

Fonte: Os autores (2021).

Tabela 2 - Estações da RBMC utilizadas com os respectivos quantitativos de dados. *DOY (Dia do ano).

\begin{tabular}{c|c|c|c|c|c}
\hline Estações RBMC & Município & Data inicial & $\begin{array}{c}\text { Total de } \\
\text { dados (Dias) }\end{array}$ & $\begin{array}{c}\text { Dados faltantes } \\
\text { (Dias) }\end{array}$ & $\begin{array}{c}\text { Dados válidos } \\
\text { (Dias) }\end{array}$ \\
\hline AMBC & Barcelos (AM) & $27 / 05 / 2015$ (DOY n 147) & 1679 & 620 & 1059 \\
AMCO & Coari (AM) & $12 / 09 / 2012$ (DOY n 256) & 2684 & 351 & 2333 \\
AMHA & Humaitá (AM) & $01 / 01 / 2015$ (DOY n 001) & 1825 & 695 & 1130 \\
AMMU & Maués (AM) & $03 / 07 / 2015$ (DOY n 184) & 1642 & 907 & 735 \\
AMTE & Tefé (AM) & $21 / 10 / 2013$ (DOY n 294) & 2262 & 340 & 1922 \\
AMUA & Manaus - UEA (AM) & $20 / 02 / 2015$ (DOY n 051) & 1775 & 309 & 1466 \\
ITAM & Itacoatiara (AM) & $30 / 10 / 2014$ (DOY n 303) & 1888 & 837 & 1051 \\
NAUS & Manaus (AM) & $01 / 01 / 2010$ (DOY no 001) & 3652 & 285 & 3367 \\
PAIT & Itaituba (PA) & $07 / 04 / 2010$ (DOY n 097) & 3555 & 604 & 2951 \\
\hline
\end{tabular}

Fonte: Os autores (2021).

Salienta-se que a área de estudo considerada está sob forte influência da carga hidrológica, característica predominante na região. Contudo, o efeito da carga hidrológica pode ser mais evidente em locais de grande concentração de massa d'água, associados às zonas úmidas e de remanso, como em Manaus. Nesse sentido, Bevis et al. (2005) utilizam a estação MANA (atual NAUS) para avaliar a propagação do efeito da carga hidrológica em seu entorno, justificando assim que tal propagação é mais significativa em um raio de até 200 quilômetros da estação considerada. Adicionalmente, Costa, Matos e Blitzkow (2012) destacam que este efeito é perceptível à distância de até 1000 quilômetros de Manaus, porém com menor amplitude. Diante disso, optou-se por utilizar nesse trabalho a estação NAUS como referência, cujas distâncias aproximadas entre esta e as demais estações são apresentadas na Tabela 3. 
Tabela 3 - Distâncias aproximadas entre estações.

\begin{tabular}{c|c|c}
\hline Estação de origem & Estação de destino & Distância aproximada (Km) \\
\hline \multirow{3}{*}{ NAUS } & AMBC & 391,91 \\
& AMTE & 518,26 \\
& AMCO & 620,88 \\
& AMHA & 596,27 \\
& AMUA & 008,65 \\
& ITAM & 180,87 \\
& AMMU & 264,05 \\
& PAIT & 467,83 \\
\hline
\end{tabular}

Fonte: Os autores (2021).

Foram utilizados no processamento arquivos de observações GNSS (GPS e GLONASS), com janelas de rastreio de 24 horas em modo estático. Contudo, o IBGE-PPP adotou a integração GPS e GLONASS no processamento em 14 de agosto de 2011, ou seja, observações referentes a datas anteriores consideram somente a constelação GPS no processamento (IBGE, 2017). No entanto, a diferença entre os resultados do processamento de dados GNSS e somente GPS é milimétrica para a seção de rastreio utilizada (YIGIT et al., 2014; VENTORIM; DAL POZ, 2016; ALCAY; YIGIT, 2017). Adicionalmente, Oliveira, Dal Poz e Almeida (2019) e Braga e Dal Poz (2019) afirmam que o IBGE-PPP apresenta acurácia análoga ao Bernese GNSS Software (versão 5.2) (FRIDEZ, 2019), considerando o método PPP em modo estático e arquivos GNSS com janela de rastreio de $24 \mathrm{~h}$.

Tabela 4 - Parâmetros de transformação entre as materializações dos ITRF (IGS). *mas: milisegundo de arco.

\begin{tabular}{c|c|c|c|c|c|c|c}
\hline Parâmetros & $\begin{array}{c}\boldsymbol{T}_{\boldsymbol{X}} \\
(\mathbf{m m})\end{array}$ & $\begin{array}{c}\boldsymbol{T}_{\boldsymbol{Y}} \\
(\mathbf{m m})\end{array}$ & $\begin{array}{c}\boldsymbol{T}_{\boldsymbol{Z}} \\
(\mathbf{m m})\end{array}$ & $\begin{array}{c}\boldsymbol{S} \\
(\mathbf{p p b})\end{array}$ & $\begin{array}{c}\boldsymbol{\varepsilon}_{\boldsymbol{X}} \\
(\mathbf{m a s})\end{array}$ & $\begin{array}{c}\boldsymbol{\varepsilon}_{\boldsymbol{Y}} \\
(\mathbf{m a s})\end{array}$ & $\begin{array}{c}\boldsymbol{\varepsilon}_{\boldsymbol{Z}} \\
(\mathbf{m a s})\end{array}$ \\
\hline ITRF2014 para ITRF2008, época 2010,0 & 1,6 & 1,9 & 2,4 & $-0,02$ & 0,000 & 0,000 & 0,000 \\
ITRF2008 para ITRF2005, época 2005,0 & $-0,5$ & $-0,9$ & $-4,7$ & 0,94 & 0,000 & 0,000 & 0,000 \\
ITRF2014 para ITRF2005, época 2010,0 & 2,6 & 1,0 & $-2,3$ & 0,92 & 0,000 & 0,000 & 0,000 \\
\hline & $\dot{\boldsymbol{T}}_{\boldsymbol{X}}$ & $\dot{\boldsymbol{T}}_{\boldsymbol{Y}}$ & $\dot{\boldsymbol{T}}_{\boldsymbol{Z}}$ & $\dot{\boldsymbol{S}}$ & $\dot{\boldsymbol{\varepsilon}}_{\boldsymbol{X}}$ & $\dot{\boldsymbol{\varepsilon}}_{\boldsymbol{Y}}$ & $\dot{\boldsymbol{\varepsilon}}_{\boldsymbol{Z}}$ \\
Variações temporais (rates) & $\mathbf{( m m}$ & $\mathbf{( m m}$ & $(\mathbf{m m}$ & $(\mathbf{p p b}$ & $(\mathbf{m a s}$ & $\mathbf{( m a s}$ & $(\mathbf{m a s}$ \\
& $/ \mathbf{a n o})$ & $/ \mathbf{a n o})$ & $/ \mathbf{a n o})$ & $/ \mathbf{a n o})$ & $/ \mathbf{a n o})$ & $/ \mathbf{a n o})$ & $/ \mathbf{a n o})$ \\
\hline ITRF2014 para ITRF2008, época 2010,0 & 0,0 & 0,0 & $-0,1$ & 0,03 & 0,000 & 0,000 & 0,000 \\
ITRF2008 para ITRF2005, época 2005,0 & 0,3 & 0,0 & 0,0 & 0,00 & 0,000 & 0,000 & 0,000 \\
ITRF2014 para ITRF2005, época 2010,0 & 0,3 & 0,0 & $-0,1$ & 0,03 & 0,000 & 0,000 & 0,000 \\
\hline
\end{tabular}

Fonte: Altamimi et. al (2007); Altamimi et. al (2012) e Altamimi et. al (2016).

As séries temporais posicionadas geradas compreenderam, no mínimo, duas materializações do ITRF (IGS). Nesse caso, compatibilizou-se as materializações ITRF2005 (IGS05) e ITRF2008 (IGS08, IGb08) para a materialização corrente (em 10/01/20), denominada ITRF2014 (IGS14), aplicando-se a transformação de Helmert com os conjuntos parâmetros de transformação descritos na Tabela 4. Salienta-se que o sentido da mudança dos referenciais deve ser cuidadosamente verificado, pois implica na alteração do sinal dos parâmetros de transformação inseridos nos modelos matemáticos.

Duas estratégias distintas foram utilizadas na compatibilização entre o ITRF2005 (IGS05) e ITRF2014 (IGS14): 1) de ITRF2005 (IGS05) para ITRF2008 (IGS08) e deste para ITRF2014 (IGS14); 2) de ITRF2005 (IGS05) diretamente para ITRF2014 (IGS14). O ITRF2008 (IGS08, IGb08) foi diretamente compatibilizado para o ITRF2014 (IGS14). Como destacado anteriormente, o IGb08 consiste em uma atualização do IGS08.

As séries temporais posicionais referenciadas ao ITRF2014 (IGS14) foram convertidas para SIRGAS2000 aplicando-se a transformação de Helmert com o conjunto parâmetros de transformação apresentados na Tabela 5. Pode-se observar que o IBGE não fornece as variações temporais dos parâmetros de transformação, impossibilitando sua atualização para a época dos dados. Além disso, salienta-se que a partir de 17 de maio de 2020 um novo conjunto de parâmetros passou a ser utilizado pelo IBGE-PPP. 
Tabela 5 - Parâmetros de transformação entre ITRF (IGS) e SIRGAS2000. *mas: milisegundo de arc.

\begin{tabular}{c|c|c|c|c|c|c|c}
\hline Parâmetros & $\begin{array}{c}\boldsymbol{T}_{\boldsymbol{X}} \\
(\mathbf{m m})\end{array}$ & $\begin{array}{c}\boldsymbol{T}_{\boldsymbol{Y}} \\
(\mathbf{m m})\end{array}$ & $\begin{array}{c}\boldsymbol{T}_{\boldsymbol{Z}} \\
(\mathbf{m m})\end{array}$ & $\begin{array}{c}\boldsymbol{S} \\
(\mathbf{p p b})\end{array}$ & $\begin{array}{c}\boldsymbol{\varepsilon}_{\boldsymbol{X}} \\
(\mathbf{m a s})\end{array}$ & $\begin{array}{c}\boldsymbol{\varepsilon}_{\boldsymbol{Y}} \\
(\mathbf{m a s})\end{array}$ & $\begin{array}{c}\boldsymbol{\varepsilon}_{Z} \\
(\mathbf{m a s})\end{array}$ \\
\hline ITRF2005 (IGS05) para SIRGAS2000 & 5,1 & 6,5 & 9,9 & 0,00 & $-0,150$ & 0,020 & 0,021 \\
ITRF2008 (IGS08) para SIRGAS2000 & 2,5 & 4,3 & 4,6 & $-1,10$ & 0,140 & $-0,010$ & 0,080 \\
ITRF2008 (IGb08) para SIRGAS2000 & 2,0 & 4,1 & 3,9 & $-1,00$ & 0,170 & $-0,030$ & 0,070 \\
ITRF2014 (IGS14) para SIRGAS2000 & 2,6 & 1,8 & $-6,1$ & $-0,05$ & 0,308 & 0,106 & $-0,096$ \\
\hline
\end{tabular}

Fonte: IBGE (2017).

As séries de componentes cartesianas geocêntricas compatibilizadas para o ITRF2014 (IGS14) e em SIRGAS2000 disponibilizadas pelo IBGE-PPP, na época dos dados, foram transformadas em componentes geodésicas locais $\Delta n, \Delta e, \Delta u$ com aplicação do modelo matemático descrito na Eq. (1). Em seguida, determinou-se as amplitudes anuais e médias das séries. Um filtro de médias móveis centrada foi aplicado às séries para suavizar as flutuações (ruído aleatório) oriundas dos erros aleatórios gerados no processamento dos dados GNSS, tornando assim a trajetória da série mais coerente à determinação das amplitudes.

A avaliação do efeito da carga hidrológica nas componentes posicionais fundamentou-se na comparação entre variações temporais de cotas linimétricas (nível do rio) e as séries de componentes $\Delta n, \Delta e$, $\Delta u$, conforme utilizado por Bevis et al. (2005), Rosa (2008), Costa, Matos e Blitzkow (2012), Giacometti et al. (2017), Nascimento et al. (2017) e Oliveira (2018). Contudo, destaca-se que a série de cotas linimétricas representa a variação superficial da água, não refletindo outros fatores relativos à carga hidrológica (como a chuva, evaporação, infiltração e os escoamentos superficial e subterrâneo), movimentos tectônicos (SAADI et al., 2002), suspensão/transporte/deposição de sedimentos nos rios (FILIZOLA; GUYOT, 2011), abalos sísmicos (ASSUMPÇÃO et al., 2014). Portanto, tal comparação não está relacionada simplesmente às magnitudes, mas ao comportamento do sinal hidrológico representado nas séries, possibilitando assim analisar seus deslocamentos de fase e amplitude (ANDERSEN et al., 2008). Nesse sentido, utilizou-se dados linimétricos coletados na estação do porto de Manaus, disponíveis em PM (2020).

Por fim, ajustou-se modelos de regressão linear simples às séries $\Delta n, \Delta e, \Delta u$, segundo a Eq. (2), para a determinação dos coeficientes de regressão $b_{1}$ e, consequentemente, das componentes de velocidades $\left(V_{n}, V_{e}, V_{u}\right)$ para cada uma das estações utilizadas. Cabe evidenciar que, como SIRGAS (2020) não especifica critérios e/ou metodologias, não foi aplicado tratamento prévio aos dados referentes às séries temporais.

Ademais, as componentes de velocidades também foram extraídas do VEMOS2009. Como suas velocidades são referenciadas ao ITRF2005 (IGS05), foram compatibilizadas para ITRF2014 (IGS14) e transformadas para o SGL conforme Carvalho (2015). As componentes de velocidade referentes à rede SIRGAS-CON (Rede SIRGAS de Monitoramento Contínuo), referenciadas ao ITRF2014 (IGS14) e no SGL foram obtidas em SIRGAS (2020a).

As etapas metodológicas anteriormente descritas são apresentadas, de forma resumida, na Figura 2. 
Figura 2 - Fluxograma das etapas metodológicas desenvolvidas.

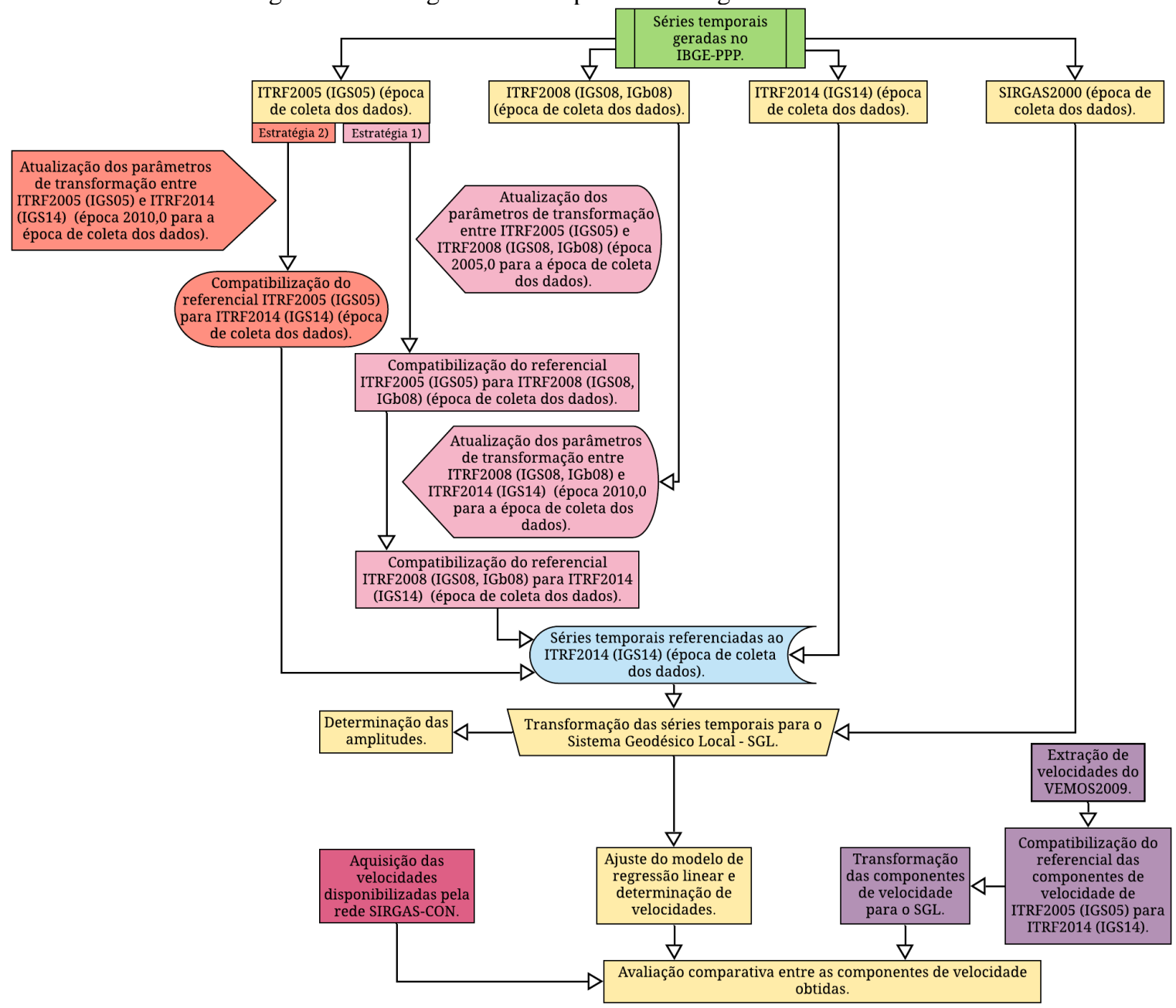

Fonte: Os autores (2021).

Ressalta-se que todos os procedimentos estatístico-matemáticos inerentes ao desenvolvimento metodológico, bem como os gráficos gerados, foram implementados em scripts no software R. Tal software caracteriza-se como uma ferramenta robusta para tais aplicações, disponibilizada gratuitamente por R Core Team (2019).

\section{RESULTADOS E DISCUSSÕES}

Uma vez que SIRGAS (2020) recomenda que as séries temporais posicionais devem originar-se de levantamentos GNSS, contínuos ou repetitivos, que cubram um período mínimo de dois anos, verificou-se que os conjuntos de dados utilizados não apresentam períodos menores que 2 anos.

Considerando as duas estratégias utilizadas para a compatibilização entre os referenciais ITRF2005 (IGS05) e ITRF2014 (IGS14), observou-se que ambas apresentaram resultados similares. Nesse caso, a segunda estratégia pode ser considerada mais viável, uma vez que reduz o esforço computacional e de implementação.

Quanto ao referencial a ser utilizado nas séries temporais, inicialmente optou-se por utilizar as séries de componentes geodésicas locais derivadas das posições geocêntricas em SIRGAS2000, disponibilizadas pelo IBGE-PPP à época do processamento dos dados (2017 a 2018). No entanto, verificou-se que as séries de componentes norte $(\Delta n)$ apresentaram uma descontinuidade de aproximadamente $0,020 \mathrm{~m}(\cong 2 \mathrm{~cm})$ referente à materialização do ITRF2014 (IGS14), degradando as velocidades $V_{n}$ referenciadas ao SIRGAS2000, principalmente para séries menores, como se pode observar na Figura 3. Ademais, as séries de componentes leste $(\Delta e)$ e vertical $(\Delta u)$ não apresentaram tal descontinuidade. 
Figura 3 - Séries de componentes norte $(\Delta n)$ derivadas das soluções em SIRGAS2000 disponibilizadas pelo IBGE-PPP.

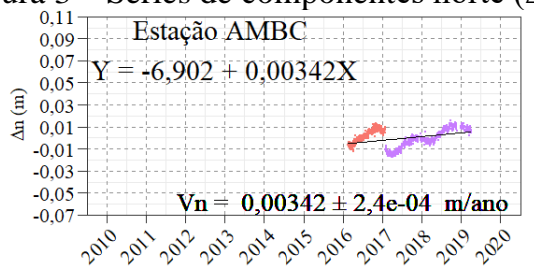

Tempo (épocas)
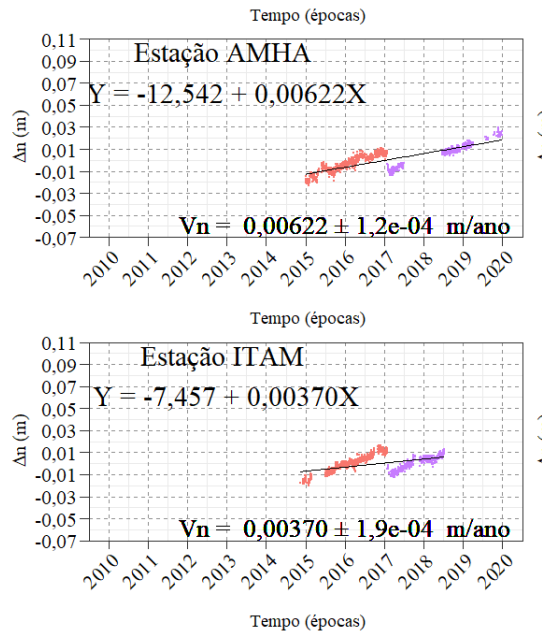

Tempo (épocas)

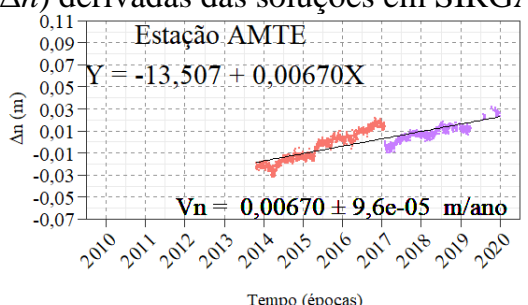

Tempo (épocas)
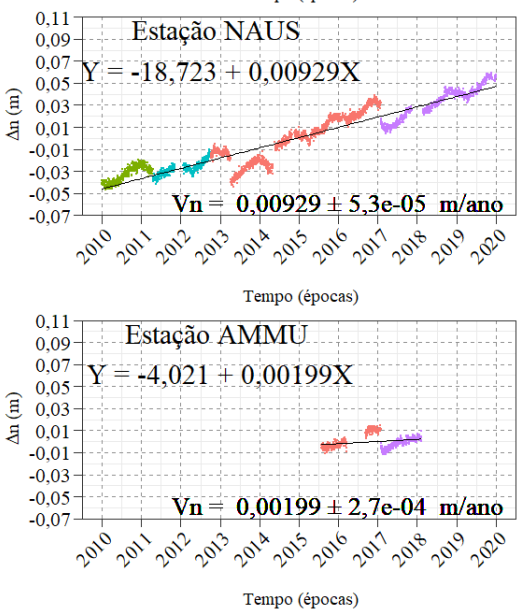

SISTEMAS DE REFERÊNCIA ORIGINAIS

- (IGS05) (IGS08) (IGb08) (IGS14)

Fonte: Os autores (2021).
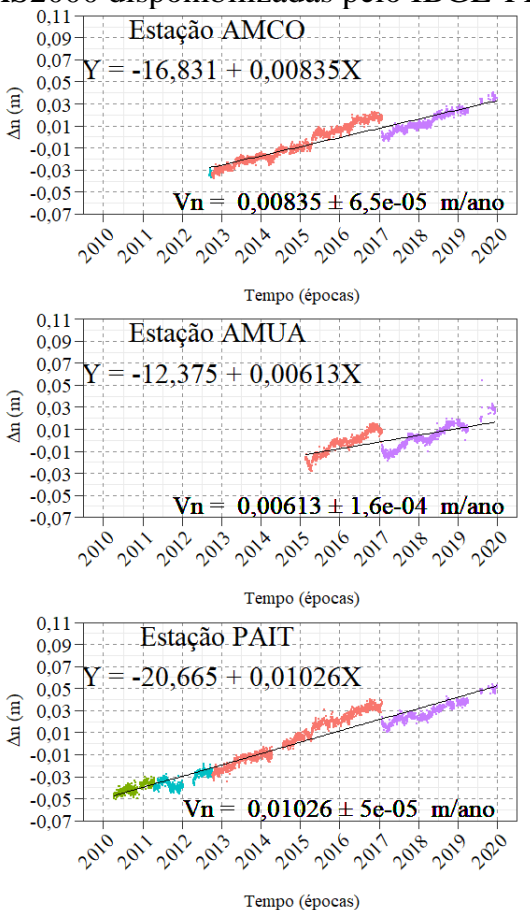

Tal fato pode ser ocasionado por fatores ligados a conversão das posições ITRF (IGS) para o SIRGAS2000, tais como: a aplicação de parâmetros de transformação "fixos", indispondo das respectivas variações temporais (ver Tabela 4), o que impossibilita sua atualização para época de destino (t); a conversão individual (direta) entre cada materialização do ITRF (IGS) e o SIRGAS2000, ou seja, de \{ITRF2005 (IGS05), ITRF2008 (IGS08), ITRF2008 (IGb08), ITRF2014 (IGS14)\} para SIRGAS2000; e/ou a maior consistência do ITRF2014 (IGS14) em relação as materializações anteriores, como descrito em Altamimi et al. (2016).

Contudo, IBGE-PPP (2020) destaca que a partir de 17 de maio de 2020 passou a adotar novos conjuntos de parâmetros de transformação entre os referenciais ITRF (IGS) e SIRGAS2000. Tal alteração visa diminuir os impactos causados pelas trocas de referenciais ocorridas ao longo do tempo. Destaca também que as diferenças entre as coordenadas determinadas com os parâmetros novos e antigos podem variar de poucos milímetros a $2 \mathrm{~cm}$ e a $3 \mathrm{~cm}$ para as componentes planimétricas e altimétricas, respectivamente. Nesse sentido, os impactos desta mudança devem ser avaliados para as séries temporais posicionais referenciadas ao SIRGAS2000 disponibilizadas pelo IBGE-PPP.

$\mathrm{Na}$ sequência, avaliou-se as séries das componentes compatibilizadas e referenciadas ao ITRF2014 (IGS14), constatando-se que estas não apresentaram tal descontinuidade e, portanto, optou-se por utiliza-las neste estudo. 
Figura 4 - Série de cotas linimétricas da estação do porto de Manaus e séries de componentes vertical $(\Delta u)$ derivadas das soluções ITRF (IGS) compatibilizadas para o ITRF2014 (IGS14).

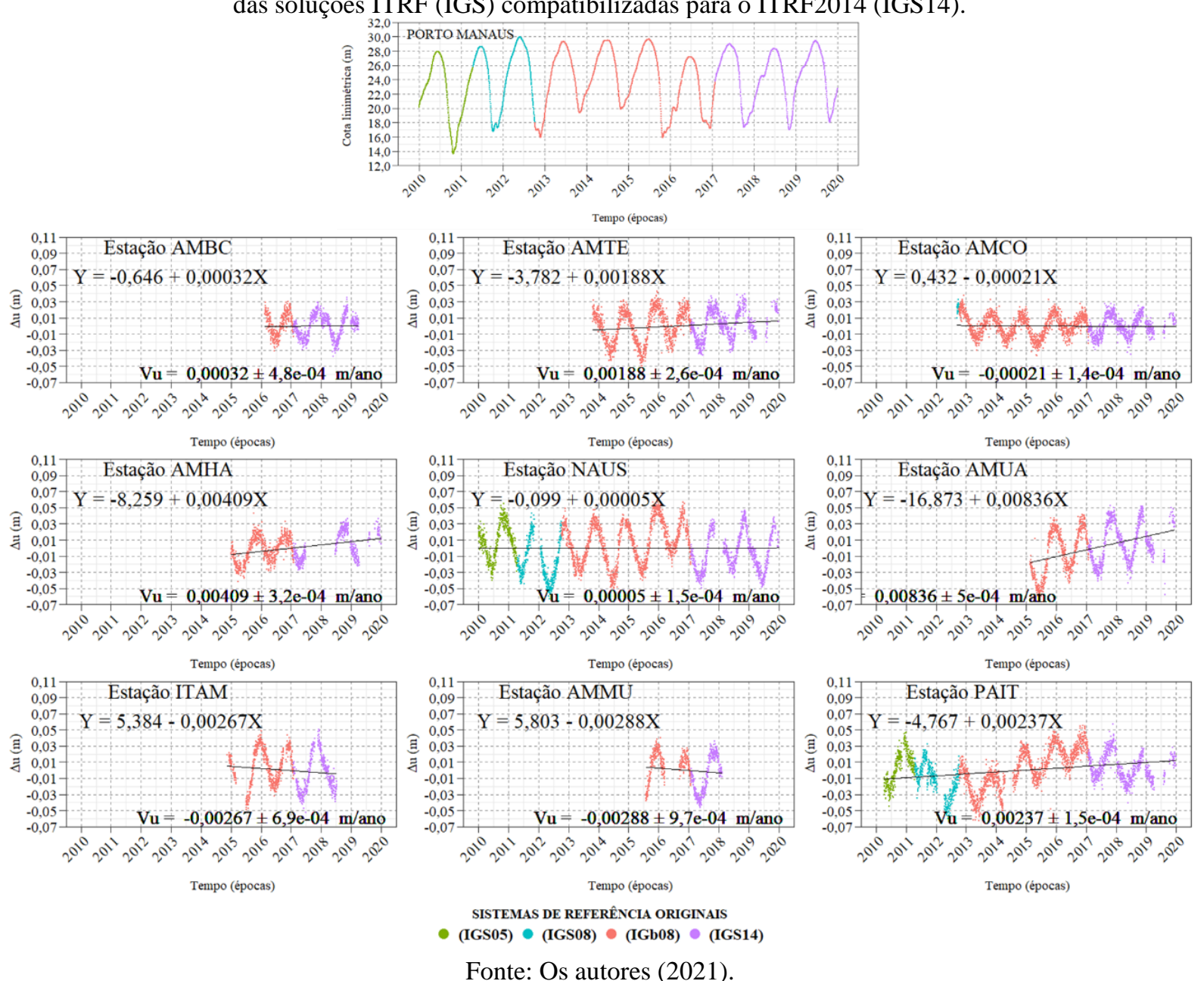

Considerando a Figura 4 pode-se observar que as séries de componentes $\Delta u$ sofrem grande influência do efeito da carga hidrológica, apresentando comportamento inverso ao da série de cotas linimétricas, com diferença de fase de aproximadamente $180^{\circ}$, reafirmando o já apresentado em Davis et al. (2004), Bevis et al. (2005) e Costa, Matos e Blitzkow (2012). Todavia, verifica-se nas Figuras 5 e 6 que as séries de componentes $\Delta n$ e $\Delta e$ também sofrem tal efeito, porém em menor grau, quando comparadas à componente vertical $\mathrm{e}$ linimétrica. 
Figura 5 - Série de cotas linimétricas da estação do porto de Manaus e séries de componentes norte $(\Delta n)$ derivadas das soluções ITRF (IGS) compatibilizadas para o ITRF2014 (IGS14).

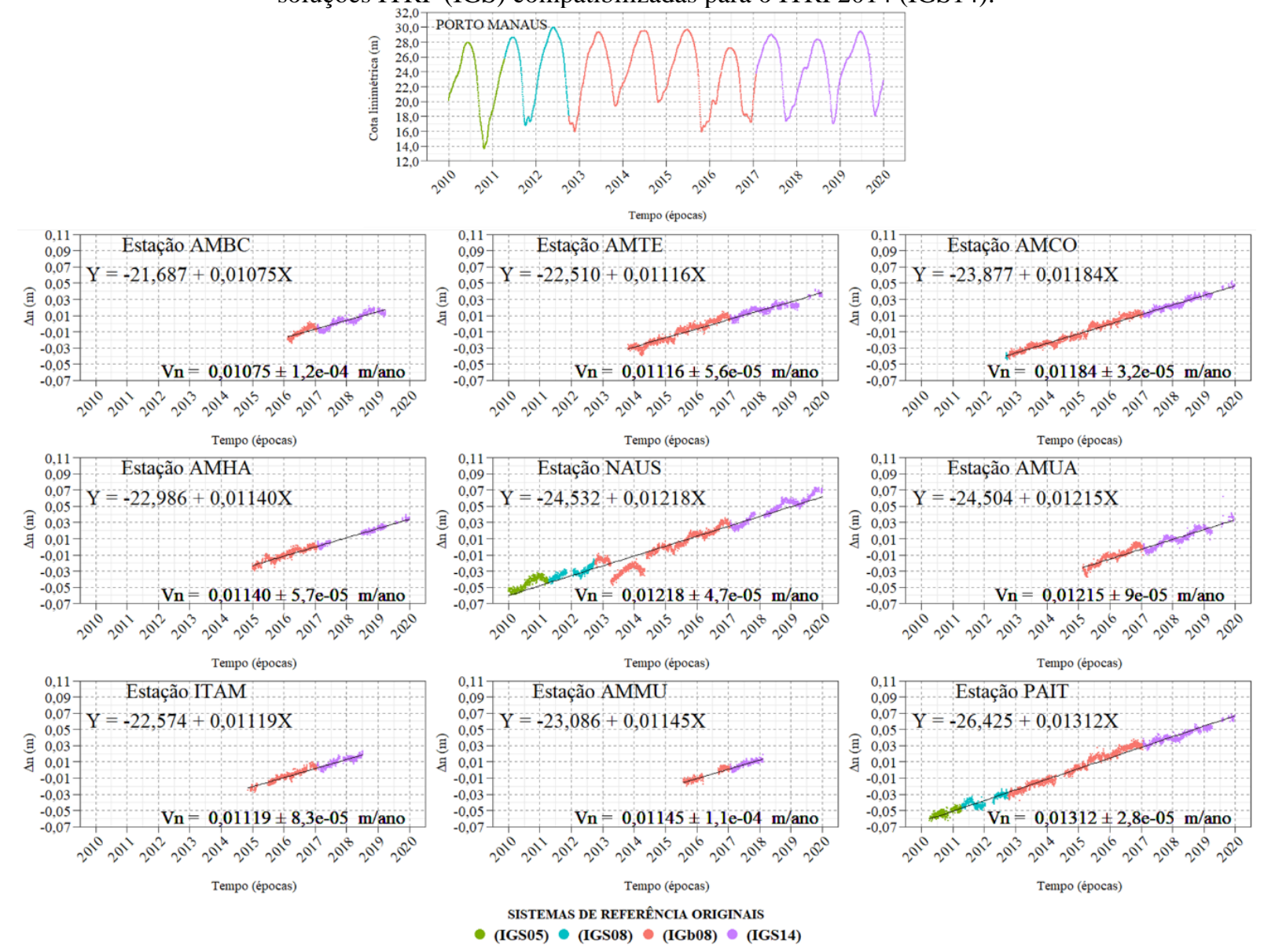

Fonte: Os autores (2021). 
Figura 6 - Série de cotas linimétricas da estação do porto de Manaus e séries de componentes este $(\Delta e)$ derivadas das soluções ITRF (IGS) compatibilizadas para o ITRF2014 (IGS14).

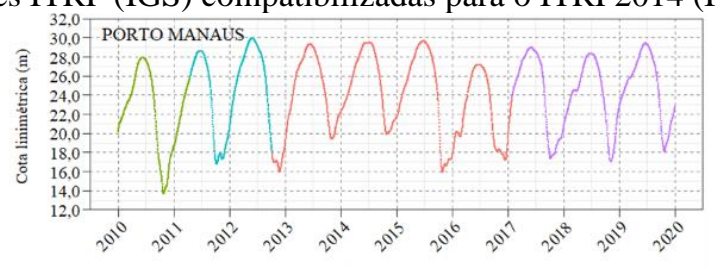

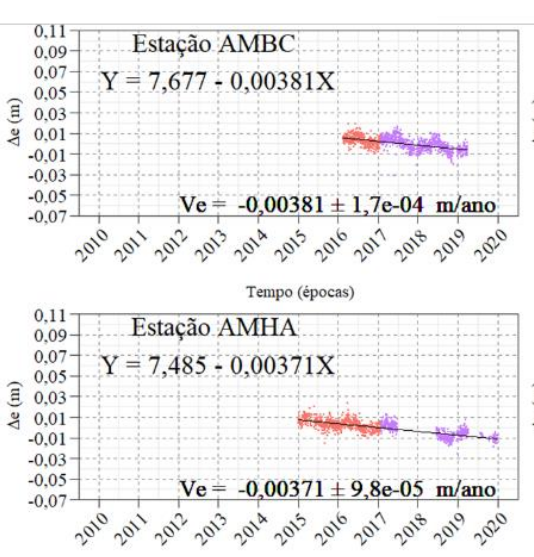

Tempo (épocas)

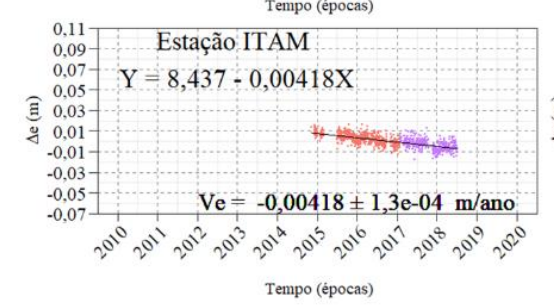

Tempo (épocas)

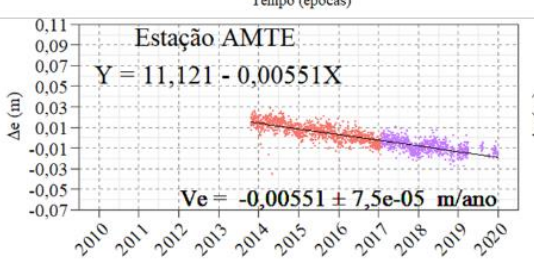

Tempo (épocas)

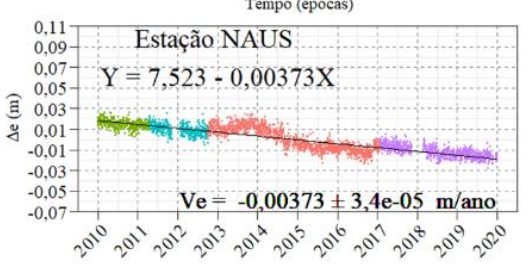

Tempo (épocas)

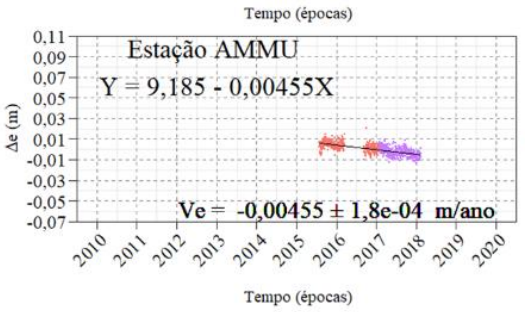

SISTEMAS DE REFERÊNCIA ORIGINAIS

- (IGS05) • (IGS08) • (IGb08) • (IGS14)

Fonte: Os autores (2021).
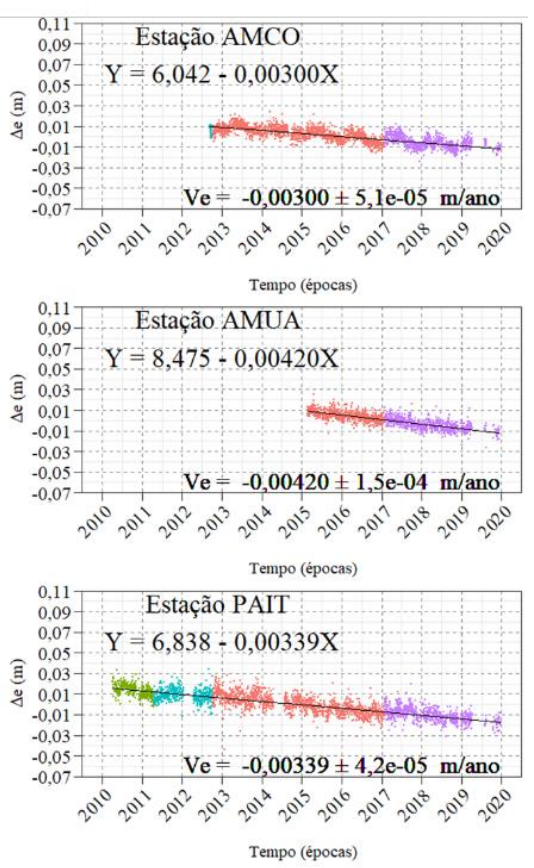

De fato, conforme Figura 7, a série $\Delta u$ da estação NAUS apresentou maior amplitude média dentre as estações consideradas. No entanto, suas maiores amplitudes ocorreram em 2012 (0,084 m) e 2015 (0,079 m), justificável pelos eventos extremos máximos (cheias), evidenciados na série de cotas linimétricas, refletindo o efeito da carga. As demais estações obtiveram amplitude média menor que a apresentada na NAUS. No entanto, a amplitude média obtida em AMUA foi similar à obtida na estação NAUS, fato justificável pela proximidade entre ambas as estações. Adicionalmente, observou-se que mesmo com maiores distâncias relativas à NAUS, as estações AMTE e AMHA não apresentaram menores amplitudes médias nas séries $\Delta u$, quando comparadas às estações AMBC e PAIT, com menores distâncias.

Analogamente à componente $\Delta u$, segundo Figura 7, NAUS apresentou maior amplitude média para série $\Delta n$, porém com valor inferior ao obtido na série $\Delta u$. A maior amplitude da série $\Delta n$ da estação NAUS também ocorreu em 2012 (0,020 m), o que mostra a influência do efeito da carga nesta componente. Salientase que as séries $\Delta n$ e $\Delta u$ das estações NAUS, AMUA e AMBC estão aproximadamente em fase. 
Figura 7 - Estatísticas referentes às amplitudes associadas às séries temporais das estações utilizadas.

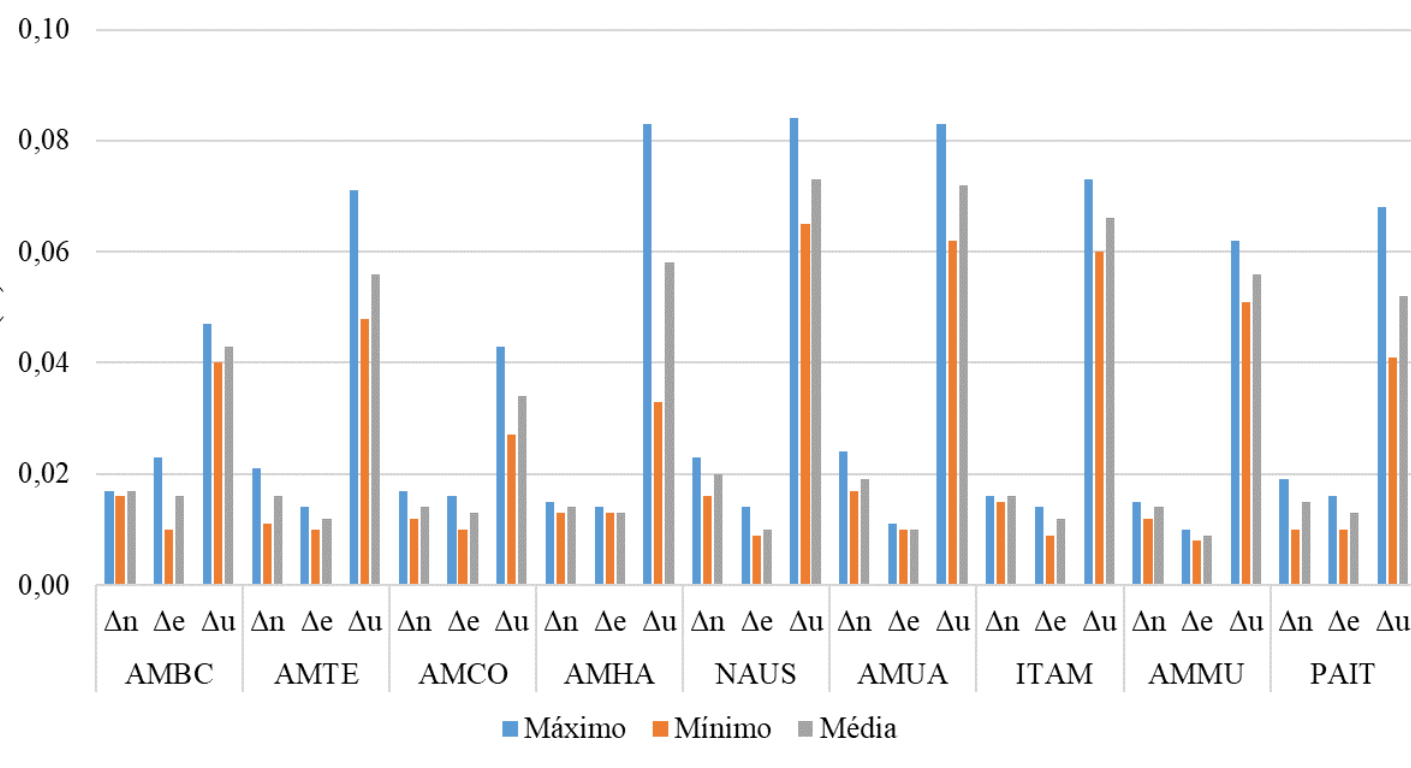

Fonte: Os autores (2021).

Em relação às séries $\Delta e$, maior amplitude média foi obtida por AMBC. Além disso, as séries $\Delta e$ das estações AMBC, AMTE e AMCO se apresentam (ou estão) fora de fase (aproximadamente $180^{\circ}$ ) em relação às séries $\Delta n$ e $\Delta u$, e em fase (aproximadamente $0^{\circ}$ ) em relação à série de cotas linimétricas.

Destaca-se que a estação NAUS apresentou uma descontinuidade (ocasionada por trocas de antenas) entre os anos de 2013 e 2014 e, portanto, desconsiderou-se este período na determinação das amplitudes.

Em virtude da comprovação da sensibilidade das séries de componentes posicionais ao efeito da carga hidrológica, pode-se inferir que as velocidades determinadas a partir destas podem ter suas estimativas mais realistas, o que justifica a recomendação de SIRGAS (2020). Nesse sentido, considerando a frequente utilização do VEMOS2009 (DREWES; HEIDBACH, 2012) para extração das velocidades no Brasil, comparou-se seus resultados aos obtidos com as séries temporais. Ademais, as velocidades determinadas a partir das séries de componentes posicionais também foram comparadas às disponibilizadas para algumas estações que integram a rede SIRGAS-CON SIRGAS (2020a).

Evidencia-se que o VEMOS2009 consiste em um modelo que representa as médias anuais dos movimentos horizontais da superfície (planimétrico), abrangendo apenas um determinado período, desconsiderando os efeitos cossísmicos (saltos) (SIRGAS, 2020). Outro fator é a baixa densidade de estações utilizadas na construção do VEMOS2009 para a região de estudo e, portanto, seus resultados não refletem o efeito da carga hidrológica, por se tratar de um modelo interpolado. Adicionalmente, a indisponibilidade das componentes verticais está associada à dificuldade da modelagem das variações verticais superficiais em nível regional, decorrentes, na maioria dos casos, de movimentos locais (IBGE, 2015).

Salienta-se que os períodos utilizados nas séries temporais abrangem eventos extremos (secas e cheias, observáveis na série linimétrica) e diferem, tanto do período de cobertura do VEMOS2009, quanto do período utilizado para a determinação das componentes disponibilizadas por SIRGAS (2020a). Ramos, Dal Poz e Carvalho (2016) ressaltam que o método de determinação das velocidades pode gerar impacto centimétrico no PPP, devido à aplicação destas nos procedimentos de atualização/redução de coordenadas.

Nesse contexto, a Tabela 6 apresenta as velocidades $\mathrm{V} n$, Ve e $\mathrm{V} u$ oriundas das séries temporais; derivadas do VEMOS2009 e fornecidas por SIRGAS (2020a). 
Tabela 6 - Componentes de velocidades referenciadas ao ITRF2014 (IGS14) determinadas por séries temporais, derivadas do VEMOS2009 e disponibilizadas pela rede SIRGAS-CON. *ND (Não disponível).

\begin{tabular}{|c|c|c|c|c|c|c|c|c|}
\hline \multirow[b]{2}{*}{ Estação RBMC } & \multicolumn{3}{|c|}{ Séries temporais } & \multicolumn{2}{|c|}{ VEMOS2009 } & \multicolumn{3}{|c|}{ V_SIRGAS-CON } \\
\hline & $\begin{array}{c}\text { Vn } \\
(\mathbf{m} \\
\text { /ano) }\end{array}$ & $\begin{array}{c}\text { Ve } \\
(\mathbf{m} \\
\text { /ano })\end{array}$ & $\begin{array}{c}\mathbf{V u} \\
(\mathbf{m} \\
\text { /ano) }\end{array}$ & $\begin{array}{c}\text { Vn } \\
(\mathbf{m} \\
\text { /ano) }\end{array}$ & $\begin{array}{c}\text { Ve } \\
(\mathbf{m} \\
\text { /ano) }\end{array}$ & $\begin{array}{c}\text { Vn } \\
(\mathbf{m} \\
\text { /ano) }\end{array}$ & $\begin{array}{c}\text { Ve } \\
(\mathbf{m} \\
\text { /ano) }\end{array}$ & $\begin{array}{c}\mathrm{Vu} \\
(\mathbf{m} \\
\text { /ano })\end{array}$ \\
\hline AMBC & 0,0108 & $-0,0038$ & 0,0003 & 0,0116 & $-0,0041$ & ND & ND & ND \\
\hline AMCO & 0,0118 & $-0,0030$ & $-0,0002$ & 0,0113 & $-0,0030$ & 0,0116 & $-0,0040$ & $-0,0005$ \\
\hline AMHA & 0,0114 & $-0,0037$ & 0,0041 & 0,0109 & $-0,0037$ & ND & ND & ND \\
\hline AMMU & 0,0115 & $-0,0046$ & $-0,0029$ & 0,0116 & $-0,0042$ & ND & ND & ND \\
\hline AMTE & 0,0112 & $-0,0055$ & 0,0019 & 0,0113 & $-0,0034$ & 0,0128 & 0,0057 & 0,011 \\
\hline AMUA & 0,0122 & $-0,0042$ & 0,0084 & 0,0112 & $-0,0054$ & ND & ND & ND \\
\hline ITAM & 0,0112 & $-0,0042$ & $-0,0027$ & 0,0115 & $-0,0044$ & ND & ND & ND \\
\hline NAUS & 0,0122 & $-0,0037$ & 0,0000 & 0,0112 & $-0,0054$ & 0,0132 & $-0,0038$ & 0,0094 \\
\hline PAIT & 0,0131 & $-0,0034$ & 0,0024 & 0,0117 & $-0,0040$ & 0,0124 & $-0,0035$ & 0,0055 \\
\hline
\end{tabular}

Fonte: Os autores (2021).

A partir das discrepâncias $\Delta \mathrm{V} n, \Delta \mathrm{V} e$ e $\Delta \mathrm{V} u$ apresentadas na Tabela 7 pode-se inferir que as variações entre as componentes determinadas com as séries temporais e as derivadas do VEMOS2009 foram de ordem submilimétrica para a maioria das estações, exceto para a componente Ve das estações AMTE e AMUA, componente $\mathrm{V} n$ da estação PAIT e componentes $\mathrm{V} n$ e $\mathrm{V} e$ da estação NAUS que apresentaram ordem milimétrica.

Tabela 7 - Discrepâncias referentes às componentes de velocidades determinadas por séries temporais, derivadas do VEMOS2009 e disponibilizadas pela rede SIRGAS-CON. *ND (Não disponível).

\begin{tabular}{|c|c|c|c|c|c|c|c|c|c|}
\hline \multicolumn{10}{|c|}{ Discrepâncias Séries temporais - VEMOS2009 } \\
\hline & AMBC & AMCO & AMHA & AMMU & AMTE & AMUA & ITAM & NAUS & PAIT \\
\hline$\Delta V \mathbf{V}(\mathrm{m} / \mathbf{a n o})$ & $-0,0009$ & 0,0005 & 0,0005 & $-0,0001$ & $-0,0001$ & 0,0009 & $-0,0003$ & 0,0010 & 0,0014 \\
\hline$\Delta V e(m / a n o)$ & 0,0003 & 0,0000 & 0,0000 & $-0,0004$ & $-0,0021$ & 0,0012 & 0,0002 & 0,0017 & 0,0006 \\
\hline \multicolumn{10}{|c|}{ Discrepâncias Séries temporais - V_SIRGAS-CON } \\
\hline & AMBC & AMCO & AMHA & AMMU & AMTE & AMUA & ITAM & NAUS & PAIT \\
\hline$\Delta V n(m / a n o)$ & ND & 0,0002 & ND & ND & $-0,0016$ & ND & ND & $-0,0010$ & 0,0007 \\
\hline$\Delta \mathrm{Ve}(\mathrm{m} / \mathrm{ano})$ & ND & 0,0010 & ND & ND & $-0,0002$ & ND & ND & 0,0001 & 0,0001 \\
\hline$\Delta V u(m / a n o)$ & ND & 0,0003 & ND & ND & $-0,0091$ & ND & ND & $-0,0094$ & $-0,0031$ \\
\hline
\end{tabular}

Fonte: Os autores (2021).

Considerando as discrepâncias referentes às componentes determinadas com as séries temporais e as disponibilizadas por SIRGAS (2020a), verificou-se que as componentes planimétricas apresentaram, em sua maioria, valores de ordem submilimétrica. No entanto, valores significativos foram observados para as discrepâncias associadas às componentes verticais, o que evidencia a necessidade de se modelar os efeitos sazonais predominantes na região em estudo para que se obtenha uma estimativa precisa das componentes de velocidade associadas a tal componente, contribuindo assim para um posicionamento preciso.

Adotando-se uma modelagem dos efeitos sazonais caracterizados pelas amplitudes observadas nas séries $\Delta u$ na determinação de $\mathrm{V} u$, destacamos que sua inserção no processo de atualização/redução de coordenadas pode (ou poderá) proporcionar resultados mais acurados. Nesse sentido, avaliar a significância das velocidades derivadas das séries temporais no processo de atualização/redução de coordenadas é de grande valia, uma vez que $\mathrm{V} u$ apresenta ordem milimétrica para maioria das estações.

\section{CONSIDERAÇÕES FINAIS, CONCLUSÕES E RECOMENDAÇÕES}

Diante do exposto pode-se constatar que as séries de componentes $\Delta n, \Delta e$ e $\Delta u$ são afetadas pelo efeito da carga hidrológica, porém, com magnitudes diferentes, influenciando assim as velocidades determinadas a partir destas, o que justifica a recomendação da utilização das séries temporais para a determinação das componentes de velocidade $\mathrm{V} n$, $\mathrm{V} e$ e $\mathrm{V} u$. 
As séries de componentes norte $(\Delta n)$ derivadas das posições em SIRGAS2000 disponibilizadas pelo IBGE-PPP apresentou descontinuidade ( $\cong 2 \mathrm{~cm}$ ) para o período de referente ao ITRF2014 (IGS14), em todas as estações utilizadas, o que pode ser associados aos parâmetros de transformação "fixos" aplicados, bem como o procedimento de conversão individual (direta) entre cada materialização do ITRF (IGS) e o SIRGAS2000. No entanto, a partir de 17 de maio de 2020, o IBGE-PPP passou a adotar novos conjuntos de parâmetros de transformação entre os referenciais, o que pode reduzir/eliminar tal descontinuidade. Os efeitos dessa mudança devem ser avaliados para as séries temporais posicionais referenciadas ao SIRGAS2000. Adicionalmente, o posicionamento de alta precisão realizado no período de transição entre o IGb08 e o IGS14, processados no IBGE-PPP antes da data supramencionada e que utilizam soluções SIRGAS2000 fornecidas pelo IBGE-PPP podem apresentar incoerências.

A expressividade do efeito de carga hidrológica é mais evidente nas séries de componentes verticais, o que pode ser observado nas amplitudes médias determinadas. No entanto, as séries de componentes planimétricas ( $\Delta n$ e $\Delta e$ ) obtiveram amplitudes médias menores quando comparadas às das componentes verticais. Observou-se que os maiores valores de amplitude média foram referentes às séries $\Delta n(0,020 \mathrm{~m}) \mathrm{e}$ $\Delta u(0,073 \mathrm{~m})$ e foram obtidos para a estação NAUS. Considerando as séries de componentes $\Delta e$ verificou-se que o maior valor de amplitude média foi apresentado na estação AMBC, atingindo 0,016 m.

Salienta-se que as séries de componentes $\Delta n$ das estações próximas ao Rio Negro (NAUS, AMUA e AMBC) apresentaram maior sensibilidade ao efeito da carga hidrológica, obtendo maior amplitude média. Além disso, observou-se que as séries $\Delta n$ e $\Delta u$ das estações NAUS, AMUA e AMBC estão aproximadamente em fase. Quando comparadas às séries de cotas linimétricas, ambas apresentaram diferença de fase de aproximadamente $180^{\circ}$. Ademais, as séries $\Delta e$ das estações AMBC, AMTE e AMCO apresentam fora de fase (aproximadamente $180^{\circ}$ ) quando comparadas às séries $\Delta n$ e $\Delta u$, e aproximadamente em fase (aproximadamente $0^{\circ}$ ) quando comparadas às séries de cotas linimétricas.

Relação inversa entre as distâncias e amplitudes não foram estabelecidas para a maioria dos casos considerados, ou seja, estações com maiores distâncias relativas à NAUS não apresentaram menores amplitudes médias. Nesse contexto, variáveis geológicas, geofísicas, sedimentares etc. devem ser consideradas nas avaliações da propagação do efeito da carga hidrológica.

Quando avaliadas as velocidades $\mathrm{V} n \mathrm{e} \mathrm{V} e$ estimadas com as séries posicionais, observou-se que estas diferiram das mesmas componentes derivadas do VEMOS2009 e das disponibilizadas por SIRGAS (2020a) e devem ter sua significância avaliada. Nesse contexto, as velocidades estimadas com as séries posicionais podem ser mais realistas por abarcarem a influência dos fatores locais, com predominância da carga hidrológica para a área considerada. Ademais, as séries temporais possibilitaram a estimação da componente $\mathrm{V} u$. Contudo, para que o usuário obtenha estimativas mais precisas para tal componente deve-se modelar os efeitos periódicos (sazonais e cíclicos) associados à componente vertical.

Por fim, recomenda-se efetuar uma análise robusta das séries temporais com a finalidade de avaliar e/ou corrigir descontinuidades artificiais, avaliar/modelar fatores periódicos e tendenciosos, o que possibilita melhores estimativas para as velocidades e deformações crustais. Além disso, abordar a determinação das velocidades com suas respectivas propagações de variâncias, com análise de precisão e acurácia também é de grande valia. Ademais, aplicar outras soluções de PPP que permita ao usuário maior domínio sobre os parâmetros de processamento é importante, pois possibilitam discretizar efeitos adicionais, permitindo uma melhor avaliação da influência da carga hidrológica.

\section{Agradecimentos}

Ao Instituto Federal do Norte de Minas (IFNMG) pela licença concedida para cursar pós-graduação e ao IBGE (Instituto Brasileiro de Geografia e Estatística) pela disponibilidade dos dados referentes à RBMC (Rede Brasileira de Monitoramento contínuo dos Sistemas GNSS) e do serviço online IBGE-PPP (Instituto Brasileiro de Geografia e Estatística - PPP). Esta pesquisa teve o incentivo da Universidade Federal de Viçosa através do Departamento de Engenharia Civil, curso de Pós-Graduação em Engenharia Civil. O presente trabalho foi realizado com apoio da Coordenação de Aperfeiçoamento de Pessoal de Nível Superior -Brasil (CAPES) - Código de Financiamento 001. 


\section{Contribuição dos Autores}

O primeiro autor foi responsável pela conceptualização, curadoria dos dados, análise formal, investigação, metodologia, software, visualização e redação - minuta inicial. O segundo autor foi responsável pela supervisão, validação e redação - revisão e edição. O terceiro autor foi responsável pela curadoria dos dados, software, visualização e redação - minuta inicial.

\section{Conflitos de Interesse}

Os autores declaram que não há conflitos de interesse.

\section{Referências}

ALCAY, S.; YIGIT, C. O. Network based performance of GPS-only and combined GPS/GLONASS positioning under different sky view conditions. Acta Geodaetica et Geophysica, v. 52, n. 3, p. 345-356, 2017. DOI: 10.1007/s40328-016-0173-5.

ALINIA, H. S.; TIAMPO, K. F.; JAMES, T. S. GPS coordinate time series measurements in Ontario and Quebec, Canada. Journal of Geodesy, v. 91, n. 6, p. 653-683, 2017. DOI: 10.1007/s00190-016-0987-5.

ALTAMIMI, Z.; COLLILIEUX, X. \& MÉTIVIER, L. Analysis and results of ITRF2008. (IERS Technical Note; 37) Frankfurt am Main: Verlag des Bundesamts für Kartographie und Geodäsie, 54 pp., ISBN 9783-86482-046-5, 2012.

ALTAMIMI, Z.; COLLILIEUX, X.; LEGRAND, J. GARAYT, B. \& BOUCHER, C. ITRF2005: A new release of the International Terrestrial Reference Frame based on time series of station positions and Earth Orientation Parameters, Journal of Geophysical Research, 112, B09401, DOI:10.1029/2007JB004949. 2007.

ALTAMIMI, Z.; REBISCHUNG, P.; MÉTIVIER, L.; COLLILIEUX, X. ITRF2014: A new release of the international terrestrial reference frame modeling nonlinear station motions. J. Geophys. Res. Solid Earth, v. 121, n. 8, p. 6109-6131, 2016. DOI: 10.1002/2016JB013098.

ANDERSEN, O., P. BERRY, J. FREEMAN, F. G. LEMOINE, S. LUTSCKHE, F. JAKOBSEN, AND M. BUTTS. Satellite altimetry and GRACE gravimetry for studies of annual water storage variations in Bangladesh. Terr. Atmos. Ocean. Sci., v. 19, p. 47-52, 2008. DOI: 10.3319/TAO.2008.19.1-2.47(SA).

ASSUMPÇÃO, M.; FERREIRA, J.M.; BEZERRA, H.; FRANÇA, G.S.; BARBOSA, J.R.; MENEZES, E.; RIBOTTA, L.C.; PIRCHINER, M.; NASCIMENTO, A.; DOURADO, J.C. Intraplate seismicity in Brazil. In: Talwani, P. Intraplate Earthquakes. S. N. A. de (Ed.). Cambridge, 2014. p. 50-71.

AZAMBUJA; J. L. F.; MATSUOKA, M. T Velocidades das coordenadas obtidas por Posicionamento por Ponto Preciso: um modelo considerando coordenadas horizontais e altura elipsoidal. Revista Brasileira de Geomática. v.4, n. 2, 2016. pp.70-80.

BEVIS, M.; ALSDORF, D.; KENDRICK, E.; FORTES, L. P.; FORSBERG, B.; SMALLEY JUNIOR, R.; BECKER, J. Seasonal fluctuations in the mass of the Amazon River system and Earth's elastic response. Geophysical Research Letters, v. 32, n. 16, pp. 1-4, 2005. DOI: 10.1029/2005GL023491.

BOGUSZ, J.; KLOS, A. On the significance of periodic signals in noise analysis of GPS station coordinates time series. GPS Solutions, v. 20, n. 4, p. 655-664, 2016. DOI: 10.1007/s10291-015-0478-9.

BRAGA, F. L. S.; DAL POZ, W. R. Potencialidades do PPP no Software Bernese com Compatibilizações de Sistemas Geodésicos de Referência e de Tempo. Rev. Bras. Cartogr., v. 71, n. 3, p. 726-755, 2019. DOI: 10.14393/rbcv71n3-47483.

BUSSAB, W. O.; MORETTIN, P. A. Estatística Básica. 9ª ed. 2017 São Paulo: Saraiva, 2005.

CARVALHO, A. S. Implicações do emprego de diferentes modelos de velocidades e parâmetros da transformação de Helmert no posicionamento geodésico na placa sul-americana. 188 p. Tese 
(Doutorado em Engenharia Civil) - Programa de Pós-Graduação em Engenharia Civil, Universidade Federal de Viçosa, Viçosa, 2015.

CARVALHO, A. S.; DAL POZ, W. R.; LAROCCA, A. P. C. Compatibilização de referenciais de coordenadas e velocidades com estimativa de precisão. Boletim de Ciências Geodésicas, Curitiba, v. 21, n. 3, p. 590609, 2015. DOI: 10.1590/S1982-21702015000300033.

CHOY, S. L; BISNATH, S.; RIZOS, C. Uncovering common misconceptions in GNSS precise point positioning and its future prospect. GPS Solutions, v. 21, n.1, pp. 13-22, 2016. DOI: 10.1007/s10291016-0545-x.

COSTA, S. M. A.; MANTOVANI, G.; SCOFANO, F.; LIMA, M. A. A.; SILVA, A. L.; MOURA JÚNIOR, N. J. Ampliação e modernização da RBMC. In: VII SIMPÓSIO BRASILEIRO DE CIÊNCIAS GEODÉSICAS E TECNOLOGIAS DA GEOINFORMAÇÃO, 2018, Recife. Anais do VII SIMGEO. p. 440-450.

COSTA, S. M. A; MATOS, A. C. O. C; BLITZKOW, D. Validation of the land water storage from gravity recovery and climate experiment (GRACE) with gauge data in the amazon basin. Boletim de Ciências Geodésicas, v. 18, n. 2, p. 262-281, 2012. DOI: 10.1590/S1982-21702012000200006.

DAVIS, J. L.; ELO'SEGUI, P..; MITROVICA, J. X.; TAMISIEA; M. E. Climate-driven deformation of the solid Earth from GRACE and GPS. Geophysical Research Letters, v. 31, L24605, 2004. DOI: 10.1029/2004GL021435.

DREWES, H.; O. HEIDBACH. The 2009 Horizontal Velocity Field for South America and the Caribbean. In: KENYON S.; PACINO M.; MARTI U. (eds). Geodesy for Planet Earth. International Association of Geodesy Symposia, Springer, Berlin, v. 136, p. 657-664, 2012. DOI: 10.1007/978-3-642-20338-1_81.

ESTEY, L. H.; MEERTENS. C. M. TEQC: The Multi-Purpose Toolkit for GPS/GLONASS Data. GPS Solutions. v. 3, n. 1, p. 42-49, 1999. DOI: 10.1007/PL00012778.

FAZILOVA, D.; EHGAMBERDIEV, SH.; KUZIN, S. Application of time series modeling to a national reference frame realization. Geodesy and Geodynamics, v. 9, p. 281-287, 2018. DOI: 10.1016/j.geog.2018.04.003.

FILIZOLA, N.; GUYOT, J. L. Fluxo de sedimentos em suspensão nos rios da Amazônia. Revista Brasileira de Geociências. v. 41, n. 4, p. 566-576, dez. de 2011. DOI: 10.25249/0375-7536.2011414566576

FRIDEZ, P. Bernese GNSS Software. Disponível em: <http://www.bernese.unibe.ch>. Acesso em: mai. 2020.

FRITSCHE, M.; DÖLL, P.; DIETRICH, R. Global-scale validation of model-based load deformation of the Earth's crust from continental watermass and atmospheric pressure variations using GPS. Journal of Geodynamics, v. 59, n. 60, p. 133- 142, 2012. DOI: 10.1016/j.jog.2011.04.001.

FU, Y.; ARGUS, D. F.; FREYMUELLER, J. T.; HEFLIN, M. B. Horizontal motion in elastic response to seasonal loading of rain water in the Amazon Basin and monsoon water in Southeast Asia observed by GPS and inferred from GRACE. Geophysical Research Letters, v. 40, 2013. DOI: 10.1002/2013GL058093.

GEIRSSON, H.; D'OREYE, N.; MASHAGIRO, N.; SYAUSWA, M.; CELLI, G.; KADUFU, B.; SMETS, B.; KERVYN, F. Volcano-tectonic deformation in the Kivu Region, Central Africa: Results from six years of continuous GNSS observations of the Kivu Geodetic Network (KivuGNet). Journal of African Earth Sciences, v. 134, p. 809-823, 2017. DOI: 10.1016/j.jafrearsci.2016.12.013.

GIACOMETTI, J. P. S.; ALBARICI, F. L.; TRABANCO, J. L. A.; GUIMARÃES, G. N. Influência da carga hídrica na altitude geométrica da estação RBMC de Inconfidentes/MG. Revista Brasileira de Geomática, v. 5, n. 1, p. 043-061, 2017. DOI: 10.3895/rbgeo.v5n1.5425.

GRINTER, T.; ROBERTS, C. Precise Point Positioning: Where are we now? In: INTERNATIONAL GLOBAL NAVIGATION SATELLITE SYSTEMS SOCIETY IGNSS SYMPOSIUM - IGNSS2011, 2011, Sydney. Proceedings of IGNSS2011. p. 15.

HOFMANN-WELLENHOF, B.; LICHTENEGGER, H.; WASLE, E. GPS Global Navigation Satellite 
Systems: GPS, GLONASS, GALILEO \& more. Austria: Springer Wien New York, 2008.

INFRAESTRUTURA NACIONAL DE DADOS ESPACIAIS (INDE). Estabelecido em 25 de fevereiro de 2015 o término do período de transição para o SIRGAS2000. Disponível em: <https://www.inde.gov.br/Noticias/Detalhe/22>. Acesso em: mai. 2020.

INSTITUTO BRASILEIRO DE GEOGRAFIA E ESTATÍSTICA (IBGE). Manual do Usuário - Aplicativo Online IBGE-PPP Versão: abril 2017. Disponível em: < https://biblioteca.ibge.gov.br/visualizacao/livros/liv101677.pdf>. Acesso em: mai. 2020.

INSTITUTO BRASILEIRO DE GEOGRAFIA E ESTATÍSTICA (IBGE). Nota Técnica. Término do período de transição para adoção no Brasil do Sistema de Referência Geocêntrico para as Américas (SIRGAS), em sua realização de 2000,4 (SIRGAS2000), 2015. Disponível em: <http://geoftp.ibge.gov.br/metodos_e_outros_documentos_de_referencia/normas/nota_tecnica_termino_ periodo_transicao_sirgas2000.pdf >. Acesso em: mai. 2020.

INSTITUTO BRASILEIRO DE GEOGRAFIA E ESTATÍSTICA-PPP (IBGE-PPP). Serviço online para pósprocessamento de dados GNSS - IBGE-PPP. Disponível em: < https://www.ibge.gov.br/geociencias/informacoes-sobre-posicionamento-geodesico/servicos-paraposicionamento-geodesico/16334-servico-online-para-pos-processamento-de-dados-gnss-ibgeppp.html?=\&t=saiba-mais-geociencias>. Acesso em: mai. 2020.

INTERNATIONAL TERRESTRIAL REFERENCE FRAME (ITRF). Call for participation. Disponível em: <http://itrf.ign.fr/doc_ITRF/CFP-ITRF2020.pdf>. Acesso em: mai. 2020.

INTERNATIONAL GNSS SERVICE ANALYSIS CENTER COORDINATOR (IGSACC). Chronology of IGS Reference Frame Usage. Disponível em: <http://acc.igs.org/igs-frames.html>. Acesso em: mai. 2020.

KAZMIER, L. J. Estatística Aplicada à Administração e Economia. 4ª ed, Porto Alegre: Bookman, 2007.

KNOWLES, L. A.; BENNETT, R. A.; HARIG C. Vertical displacements of the Amazon basin from 2 GRACE and GPS. Journal of Geophysical Research: Solid Earth, v. 125, n. 2, 2020. DOI: 10.1029/2019JB018105.

KOWALCZYK, K.; RAPINSKI, J. Verification of a GNSS Time Series Discontinuity Detection Approach in Support of the Estimation of Vertical Crustal Movements. ISPRS International Journal of GeoInformation, v. 7, n. 149, p. 1-15, 2018. DOI: 10.3390/ijgi7040149.

LEICK, A. GPS Satellite Surveying. 3rd ed. Hoboken: John Wiley and Sons, 2004.

LEVINE, D.; M. STEPHAN, D. F.; KREHBIEL, T. C.; BERENSON, M. L. Estatística-Teoria e Aplicações Usando Microsoft Excel em Português. Traduzido por Teresa Cristina Padilha de Souza, $6^{\mathbf{a}}$ ed. Rio de Janeiro: LTC, 2013.

MACIUK, K.; SZOMBARA, S. Annual crustal deformation based on GNSS observations between 1996 and 2016. Arabian Journal of Geosciences, v. 11, n. 21, p. 1-12, 2018. DOI: 10.1007/s12517-018-4022-4.

MONICO, J. F. G. Posicionamento pelo GNSS: Descrição, fundamentos e aplicações. $2^{\mathrm{a}}$.ed. São Paulo: Editora UNESP, 2008. 476 p.

MOREIRA, D. M. Rede de referência altimétrica para avaliação da altimetria por satélites e estudos hidrológicos na região amazônica. 157 p. Dissertação (Mestrado em Engenharia Civil) - Programa de Pós-graduação em Engenharia Civil, Universidade Federal do Rio de Janeiro, Rio de Janeiro, 2010.

MOREIRA, D. M., S.; CALMANT, S.; PEROSANZ, F.; XAVIER, L.; ROTUNNO FILHO, O. C.; SEYLER, F.; MONTEIRO, A. C. Comparisons of observed and modeled elastic responses to hydrological loading in the Amazon basin, Geophys. Geophysical Research Letters. v. 43, p. 262-281, 2016. DOI: 10.1002/2016GL070265.

NASCIMENTO, L. A. Influência da carga hidrológica na altitude geométrica a partir de análise de séries temporais estimadas no método PPP. 73 p. Dissertação (Mestrado em Engenharia Civil) - Programa de Pós Graduação em Engenharia Civil, Universidade Federal de Viçosa, Viçosa, 2016.

NASCIMENTO, L. A.; DAL POZ, W. R.; SANTOS, A. P.; FREITAS, K. X. S. Geração automatizada de 
séries temporais de dados posicionais utilizando o IBGE-PPP. Boletim de Ciências Geodésicas, v. 23, n. 1, p.150-165, 2017. DOI: 10.1590/S1982-21702017000100010.

NATURAL RESOURCES CANADA (NRCAN). CSRS-PPP: Canadian Spatial Reference System - Precise Point Positioning. Disponível em: <https://www.google.com/search?q=CSRS-PPP\&oq=CSRSPPP\&aqs=chrome..69i57j017.1576j0j4\&sourceid=chrome\&ie=UTF-8>. Acesso em: mai. 2020.

OLDS, S.; ERIKSSON, S.; MUNSON, D.; DAHLMAN L. Analyzing Plate Motion Using EarthScope GPS Data, 2019. Disponível em: <https://serc.carleton.edu/eet/platemotion/index.html>. Acesso em: abr. 2020.

OLIVEIRA, G. D.; DAL POZ, W. R.; ALMEIDA, M. S. Implementação de um sistema on-line para processamento de dados GNSS. Revista Brasileira de Cartografia, v. 71, n. 2, p. 501-512, 2019. DOI: $10.14393 /$ rbcv71n2-48184.

OLIVEIRA, J. R. S. Uso de informações geodésicas para estudos tectônicos no Pantanal. 59 p. Dissertação (Mestrado em Recursos Minerais e Meio Ambiente) - Programa de Pós-Graduação em Recursos Minerais e Meio Ambiente, Universidade de São Paulo, São Paulo, 2018.

PETIT, G.; LUZUM, B. IERS Conventions (2010). IERS Technical Note $\mathrm{N}^{\circ} 36$, Frankfurt am Main: Verlag des Bundesamts für Kartographie und Geodäsie, 2010. ISBN 3-89888-989-6.

PORTO DE MANAUS (PM). Nível do Rio Negro. Disponível em: < https://www.portodemanaus.com.br/?pagina=nivel-do-rio-negro-hoje>. Acesso em: 20 mai. 2020.

R CORE TEAM. R: A language and environment for statistical computing. R Foundation for Statistical Computing, Vienna, 2019. Disponível em: <https://www.R-project.org/>. Acesso em: mar. 2020.

RAJNER, M. Detection of ice mass variation using GNSS measurements at Svalbard. Journal of Geodynamics, v. 121, p. 20-25, 2018. DOI: 10.1016/j.jog.2018.06.001.

RAJNER, M.; LIWOSZ, T. Studies of crustal deformation due to hydrological loading on GPS height estimates. Geodesy and Cartography, v. 60, n. 2, p. 135- 144, 2012. DOI: 10.2478/v10277-012-0012-y.

RAMOS, M. P.; DAL POZ, W. R.; CARVALHO, A. S. Análise das possibilidades de transformação de referencial e atualização de coordenadas estimadas pelo PPP. Boletim de Ciências Geodésicas, v. 22, n. 3, p.389-404. 2016. DOI: 10.1590/S1982-21702016000300022.

REBISCHUNG, P. [IGSMAIL-6663]. IGb08: an update on IGS08. Disponível em: <https://lists.igs.org/pipermail/igsmail/2012/000497.html>. Acesso em: mai. 2020a.

REBISCHUNG, P. [IGSMAIL-7921] Switch to IGb14 reference frame. Disponível em: < https://lists.igs.org/pipermail/igsmail/2020/007917.html>. Acesso em: mai. 2020b.

REDE BRASILEIRA DE MONITORAMENTO CONTÍNUO DOS SISTEMAS GNSS (RBMC). Dados diários e situação operacional. Disponível em: <https://www.ibge.gov.br/geociencias/informacoes-sobreposicionamento-geodesico/rede-geodesica/16258-rede-brasileira-de-monitoramento-continuo-dossistemas-gnss-rbmc.html?=\&t=dados-diarios-e-situacao-operacional>. Acesso em: mai. 2020.

ROMAGNOLI, C; ZERBINI, S; LAGO, L; RICHTER, B; SIMON, D; DOMENICHINI, F; ELMI, C; GHIROTTI, M. Influence of soil consolidation and thermal expansion effects on height and gravity variations. Journal of Geodynamics, v. 35. p. 521-539, 2003. DOI: 10.1016/S0264-3707(03)00012-7.

ROSA, G. P. S. Análise de séries temporais de coordenadas estimadas com GPS: uma proposta metodológica para eliminação de efeitos sazonais. 106 p. Dissertação (Mestrado em Ciências Cartográficas) - Programa de Pós-Graduação em Ciências Cartográficas, Universidade Estadual Paulista, Presidente Prudente, 2008.

SAADI, A.; MACHETTE, M. N.; HALLER, K. M.; DART, R. L.; BRADLEY, L.; SOUZA, A. M. P. D. Map and Database of Quaternary Faults and Lineaments in Brazil. Denver: U.S. Geological Survey, June 2002. 1:6.000.000.

SÁNCHEZ, L.; DREWES H. Crustal deformation and surface kinematics after the 2010 earthquakes in Latin America. Journal of Geodynamics, United Kingdom, v. 102, p. 1-23, dez. 2016. DOI: 10.1016/j.jog.2016.06.005. 
SAPUCCI, L. F.; MONICO, J. F. G. Transformação de Helmert generalizada no posicionamento de alta precisão: fundamentação teórica e exemplificações. Brazilian Journal of Geophysics, Presidente Prudente-SP, v. 18, n. 2, p. 161-172. 2001. DOI: 10.1590/S0102-261X2000000200005.

SILVA, H. A.; MONICO, J. F. G. Análise de séries temporais de coordenadas GNSS. In: IV Simpósio Brasileiro de Ciências Geodésicas e Tecnologias da Geoinformação, 2018, Recife. Anais do IV SIMGEO. p. 001-009.

SISTEMA DE REFERÊNCIA GEOCÊNTRICO PARA AS AMÉRICAS (SIRGAS). VEMOS: Modelo de velocidade para o SIRGAS. Disponível em: <http://www.sirgas.org/pt/sirgas-con-network/velocitymodel/>. Acesso em: mai. 2020.

SISTEMA DE REFERÊNCIA GEOCÊNTRICO PARA AS AMÉRICAS (SIRGAS). SIRGAS-CON: Rede SIRGAS de monitoramento contínuo. Disponível em: <http://www.sirgas.org/pt/sirgas-connetwork/stations/>. Acesso em: mai. 2020a.

TREGONING, P.; WATSON, C.; RAMILLIEN, G.; MCQUEEN, H.; ZHANG, J. Detecting hydrologic deformation using GRACE and GPS. Geophysical Research Letters, v. 36, n. 15, p. 1-6, 2009. DOI: $10.1029 / 2009$ GL038718.

UNAVCO. TEQC - The Toolkit for GNSS Data. Disponível em:<https://www.unavco.org/software/dataprocessing/teqc/teqc.html>. Acesso em: abr. 2020.

VAN DAM, T.; WAHR, J.; MILLY, P. C. D.; SHMAKIN, A. B.; BLEWITT, G.; LAVALLÉE, D.; LARSON, K. M. Crustal displacements due to continental water loading. Geophysical Research Letters, v. 28, n. 4, p. 651-654, 2001. DOI: 10.1029/2000GL012120.

VENTORIM, B. G; DAL POZ, W. R. Avaliação do desempenho dos sistemas GPS e GLONASS no posicionamento por ponto preciso online, combinados e individualmente. Boletim de Ciências Geodésicas, Curitiba, v. 22, n. 2, p. 264-281. Abr-jun 2016. DOI: 10.1590/S1982-21702016000200015.

YIGIT, C. O.; GIKAS, V.; ALCAY, S.; CEYLAN, A. Performance evaluation of short to long term GPS, GLONASS and GPS/GLONASS postprocessed PPP. Survey Review, v. 46, n. 336, pp. 155-166, 2014. DOI: $10.1179 / 1752270613$ Y.0000000068.

\section{Biografia do autor principal}

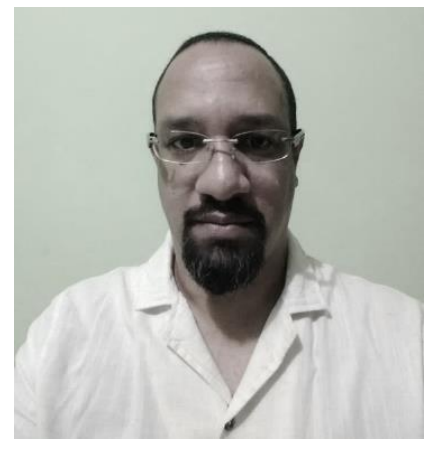

Lécio Alves Nascimento é natural de Itabuna, Bahia, Brasil. É Engenheiro Agrimensor e Cartógrafo pela Universidade Federal de Viçosa - UFV, mestre e doutor em Informações Espaciais pelo Programa de Pós-Graduação em Engenharia Civil - PPGEC da UFV, Campus Viçosa. Atualmente é professor do Ensino Básico, Técnico e Tecnológico - EBTT no Instituto Federal de Ciência e Tecnologia do Norte de Minas Gerais - IFNMG, Campus Araçuaí. Atua nas áreas relacionadas a Agrimensura e às Geociências, onde desenvolve pesquisas relacionadas à Geodésia e Programação aplicada, cujos estudos concentram-se na Geodinâmica e deformações associadas ao efeito de carga hidrológica, modelagem e análises de séries temporais. 